\title{
Tight-binding parameters for charge transfer along DNA
}

\author{
L.G.D. Hawke, ${ }^{1}$ G. Kalosakas, ${ }^{1}$ and C. Simserides ${ }^{2}$ \\ ${ }^{1}$ Materials Science Department, University of Patras, Rio GR-26504, Greece \\ ${ }^{2}$ Institute of Materials Science, National Center of Scientific Research Demokritos, GR-15310 Athens, Greece
}

\begin{abstract}
We systematically examine all the tight-binding parameters pertinent to charge transfer along DNA. The $\pi$ molecular structure of the four DNA bases (adenine, thymine, cytosine, and guanine) is investigated by using the linear combination of atomic orbitals method with a recently introduced parametrization. The HOMO and LUMO wavefunctions and energies of DNA bases are discussed and then used for calculating the corresponding wavefunctions of the two B-DNA base-pairs (adenine-thymine and guanine-cytosine). The obtained HOMO and LUMO energies of the bases are in good agreement with available experimental values. Our results are then used for estimating the complete set of charge transfer parameters between neighboring bases and also between successive base-pairs, considering all possible combinations between them, for both electrons and holes. The calculated microscopic quantities can be used in mesoscopic theoretical models of electron or hole transfer along the DNA double helix, as they provide the necessary parameters for a tight-binding phenomenological description based on the $\pi$ molecular overlap. We find that usually the hopping parameters for holes are higher in magnitude compared to the ones for electrons, which probably indicates that hole transport along DNA is more favorable than electron transport. Our findings are also compared with existing calculations from first principles.
\end{abstract}

PACS numbers: 73.63.-b, 82.39.Jn,87.14.gk, 87.15.A-

\section{INTRODUCTION}

DNA plays a fundamental role in genetics and molecular biology since its sequence of bases, adenine (A), guanine $(\mathrm{G})$, cytosine $(\mathrm{C})$, and thymine $(\mathrm{T})$, contains the genetic code of living organisms. During the last decade, DNA and its charge transport properties have attracted the interest of a large interdisciplinary community due to its potential use for nanodevices, either for assembling nanocircuits or as a molecular wire [1, 2, 3, 4]. Charge migration through DNA could also play an important role in biology; it may be a critical issue in carcinogenesis and mutagenesis [5, 6]. For example the rapid hole migration from other bases to guanine is connected to the fact that direct strand breaks occur preferentially at guanines [5]. Long-range charge transfer along the $\pi$-stacking of the DNA double helix may also be crucial for DNA damage and repair 7,8$]$.

However, it is not yet clear "how much" DNA conducts. Experiments cover a wide range of behavior. In particular, previous results found that $\lambda$-DNA covalently bonded to Au electrodes is an insulator [9]. Furthermore, insulating behavior - both for single DNA molecules as well as for small bundles of DNA molecules- was observed at the $100 \mathrm{~nm}$ length scale [10]; this was confirmed both for mixed base-pair sequence as well as for homogeneous poly $(\mathrm{dG})$-poly $(\mathrm{dC})$, for lengths between contacts in the range $40-500 \mathrm{~nm}$, for substrate $\mathrm{SiO}_{2}$ or mica, and for electrode material gold or platinum. The absence of $d c$ conductivity in $\lambda$-DNA was also reported for DNA molecules adsorbed on mica, in agreement with the authors' first principles electronic structure calculations [11].

In a different direction point the results of Yoo et al. 12], who investigated the electrical transport through poly $(\mathrm{dA})-\operatorname{poly}(\mathrm{dT})$ and poly $(\mathrm{dG})$-poly $(\mathrm{dC})$
DNA, i.e. molecules containing identical base-pairs. Their experimental results suggest that electrical transport through DNA molecules occurs by polaron hopping and the possibility of a DNA field-effect transistor operating at room temperature was also demonstrated [12]. Moreover, gate-voltage dependent transport measurements showed that poly $(\mathrm{dA})-\operatorname{poly}(\mathrm{dT})$ behaves as a n-type semiconductor, whereas poly $(\mathrm{dG})$-poly $(\mathrm{dC})$ behaves as a p-type semiconductor [12. In another report it has been shown that poly $(\mathrm{dG})$-poly $(\mathrm{dC})$ can act as a semiconducting nanowire exhibiting better conductance than poly(dA)-poly(dT) [13].

Measurements of electrical current as a function of the potential applied across single DNA ropes at least $600 \mathrm{~nm}$ long, indicated metallic-like gapless behavior and efficient conduction comparable to that of conducting polymers 14]. In this work it was mentioned that the observed behavior together with the fact that DNA molecules of specific composition and length, ranging from a few nucleotides to several tens of $\mu \mathrm{m}$, can be prepared, makes DNA ideally suited for the construction of mesoscopic electronic devices [14].

The $a c$ conductivity of DNA has been also explored. For example, $\lambda$-phage DNA at microwave frequencies showed strong temperature dependence of the $a c$ conductivity around room temperature, exhibiting a crossover to a weak temperature dependence at lower temperatures 15]. Measurements of the quasi-static, frequencydependent conductivity below $1 \mathrm{MHz}$ performed on wetspun, macroscopically oriented, calf thymus DNA bulk samples, showed that the electrical conductivity can be rather well described by an activated Arrhenius law with activation energy of $0.9 \mathrm{eV}$ or by hole hopping [16].

The observed important deviations in the conductivity of DNA can be -at least partially- attributed to several 
external conditions like the type of the substrate, the distance between the electrodes, and the contact material, in conjunction with the soft nature of the biomolecule. In addition, several intrinsic characteristics, like the local chemical environment which is a result of the solution involved in the DNA preparation [17], the hydrogen bonding [18], and the degree of stretching of DNA [19] affect the electronic properties.

The great effort to ameliorate DNA's transport properties led to alternative molecular conductive candidates based on DNA. In M-DNA the imino-proton of each basepair has been substituted with a metal ion [20]. In contrast to B-DNA, M-DNA presents no plateau in its I-V curve and exhibits metallic-like conduction at room temperature [20]. Size-expanded DNA bases are produced by the addition of a benzene ring to a natural DNA base [21] and have been combined with natural bases to form $\mathrm{xDNA}$ and $\mathrm{yDNA}$, a new class of synthetic nucleic acids. It has been theoretically shown that xDNA and yDNA have smaller HOMO-LUMO gap than B-DNA [21], i.e., they might function as molecular wires better than natural DNA.

Many mechanisms have been suggested to explain the charge transport along DNA, where generally $\pi$-pathway transfer due to the overlap of $\pi$ molecular orbitals of the stacked aromatic bases of DNA can lead to charge propagation even at long distances [4, 7, 22]. Examples of mechanisms that have been examined are band transport [23, 24], polaronic transport [12, 25, 26, 27, 28, 29], fluctuation-facilitated charge migration [30, 31, 32, 33], and variable range hopping [34].

The aim of this work is to provide electronic parameters for charge (electron or hole) transfer along DNA, assumed that the transport mechanism is the $\pi$-pathway. Using the linear combination of atomic orbitals (LCAO) method, our investigation focuses on the calculation of HOMO (highest occupied molecular orbital) and LUMO (lowest unoccupied molecular orbital) $\pi$ molecular wavefunctions and energies for the four DNA bases, A, G, C, $\mathrm{T}$, and the two B-DNA base-pairs, adenine-thymine (A$\mathrm{T}$ ) and guanine-cytosine (G-C). Then the hopping matrix elements between neighboring bases or base-pairs, for all possible combinations between them, are provided, for both electrons and holes. The obtained hopping parameters and the HOMO and LUMO energies can be used in a tight-binding phenomenological description of charge transfer along DNA. Such a mesoscopic description is applied in numerous theoretical models of charge transport [25, 26, 27, 29, 33, 34, 35, 36, 37]. Our results are compared with existing calculations from first principles, as well as with available experimental data for the HOMO and LUMO energies of DNA bases.

The article is organized in the following way: In Sec.II we explain in detail our theoretical approach. Specifically, in Sec. IIA we apply a simple LCAO method for the $\pi$ electronic structure of the four DNA bases using a novel parametrization [38], in Sec. IIB we discuss the HOMO and the LUMO of base-pairs by employing a sim- ilar linear combination of molecular orbitals method, and finally Sec. $\amalg \mathrm{CC}$ is devoted to the tight-binding description and the calculation of charge transfer parameters in this framework. In Sec. III] we present and discuss our results, along with a comparison with earlier theoretical calculations as well as with available experimental values. Finally, in Sec. IV we state our conclusions.

\section{THEORY}

\section{A. $\pi$ molecular structure of DNA bases}

In this subsection we present the LCAO methodology used for the description of the $\pi$ electronic structure of the four isolated DNA bases, A, G, C, and T. DNA bases are planar organic molecules bonded by $s p^{2}$ hybridization, where the atoms have their $p_{z}$ atomic orbitals perpendicular to the molecular plane. The electrons that occupy these atomic orbitals are delocalized to form $\pi$ molecular orbitals. The LCAO method provides a straightforward approach to obtain the $\pi$ molecular structure. In its simpler form, used in the present article, a $\pi$ molecular single-electron wavefunction can be approximated as

$$
\Psi^{b}(\mathbf{r})=\sum_{i=1}^{N} c_{i} p_{z}^{i}(\mathbf{r}) .
$$

The index $i$ implies summation over all atoms ( $N$ totally) which contribute $p_{z}$ electrons to the DNA base of interest. $\left|c_{i}\right|^{2}$ gives the probability of finding the electron occupying the molecular orbital $\Psi^{b}(\mathbf{r})$ at the atom indexed by $i$, while $p_{z}^{i}(\mathbf{r})$ denotes the corresponding atomic, or atomic-like, orbital. The molecular wavefunction obeys Schrödinger equation $H^{b} \Psi^{b}(\mathbf{r})=E^{b} \Psi^{b}(\mathbf{r})$, where $E^{b}$ is the eigenergy of the base. Substituting in the latter equation $\Psi^{b}(\mathbf{r})$ through Eq. (1), multiplication with the conjugate orbital $p_{z}^{j \star}(\mathbf{r})$, and integration over all space, gives a linear system of $N$ equations obeyed by the unknown coefficients $c_{i}$ of the molecular wavefunction under determination and its energy eigenvalue $E^{b}$. Following a standard procedure and assuming that the atomic or atomiclike $p_{z}$ orbitals located at different atoms are orthogonal, one obtains that the solution of the linear system of equations determining $c_{i}$ and the corresponding $E^{b}$, is equivalent to the diagonalization of the Hamiltonian with matrix elements $H_{i j}^{b}=\int d^{3} r p_{z}^{i \star}(\mathbf{r}) H^{b} p_{z}^{j}(\mathbf{r})$ (see for example Ref. [38]).

Through numerical diagonalization of the symmetric $N \times N$ Hamiltonian matrix $H_{i j}^{b}$ we obtain the coefficients $c_{i}$ (eigenvectors) providing the molecular orbitals $\Psi^{b}(\mathbf{r})$ [cf. Eq. (1)], as well as the corresponding eigenenergies $E^{b}$. To this end we need the values of the Hamiltonian matrix elements, $H_{j i}^{b}$. We employ a recently introduced parametrization that has been successfully used for the description of the energy of $\pi$ frontier orbitals in more than sixty planar organic molecules [38], including the 
DNA bases, as well as in flavin [39], and has shown to provide accurate calculations of the $\pi \mathrm{HOMO}$ energies and $\pi-\pi^{*}$ gaps. Regarding the diagonal matrix elements, $H_{i i}^{b} \equiv \varepsilon_{i}$, we use $\varepsilon_{C}=-6.7 \mathrm{eV}$ for carbon atoms, $\varepsilon_{N_{2}}=-7.9 \mathrm{eV}$ for nitrogen atoms contributing one $p_{z}$ electron (i.e. with coordination number 2), $\varepsilon_{N_{3}}=-10.9$ $\mathrm{eV}$ for nitrogen atoms with two $p_{z}$ electrons (i.e. with coordination number 3 ), and $\varepsilon_{O}=-11.8 \mathrm{eV}$ for oxygen atoms. For the nondiagonal matrix elements $H_{i j}^{b}(i \neq j)$ referring to neighboring $s p^{2}$-bonded atoms we use the following expression proposed by Harrison [40]:

$$
H_{i j}^{b}=V_{p p \pi}=-0.63 \frac{\hbar^{2}}{m d^{2}},
$$

where $m$ is the electron mass and $d$ is the distance between the corresponding nearest-neighboring atoms. All other nondiagonal matrix elements, referring to atoms not $s p^{2}$-bonded are assumed equal to zero, $H_{i j}^{b}=0$.

Diagonalizing the Hamiltonian matrix, one obtains $N$ molecular orbitals and their eigenenergies. The lower energy orbitals are successively filled by two electrons each, until to accommodate all available $p_{z}$ electrons. Then the highest-energy occupied orbital, $\Psi_{H}^{b}(\mathbf{r})$, is the $\pi$ HOMO and the lowest-energy unoccupied one, $\Psi_{L}^{b}(\mathbf{r})$, is the $\pi$ LUMO. Following the usual convention, we denote occupied molecular orbitals by $\pi$ and unoccupied ones by $\pi^{*}$.

\section{B. HOMO and LUMO of B-DNA base-pairs}

Regarding the B-DNA base-pairs, we follow a different procedure in order to obtain the HOMO and LUMO wavefunctions. This is because the two bases (A and T, or $\mathrm{G}$ and $\mathrm{C}$ ) are connected with non-covalent hydrogen bonds to form the base-pair (A-T or G-C, respectively). The length of hydrogen bonds, around $3 \AA$, is longer than the typical length of a covalent bond connecting neighboring atoms within a base, which is around 1.3-1.5 . In other words, a base-pair is not assumed in our calculation like a single molecule but, instead, it is treated like two adjacent molecules with electronic overlap. However, we still use the terms HOMO and LUMO for the basepairs, meaning the single-electron wavefunctions which represent the highest in energy occupied orbital and the lowest in energy unoccupied orbital, respectively, of the molecular complex. We assume that these wavefunctions describe an inserted hole or electron, respectively, within a base-pair. Below we follow a straightforward linear combination of molecular orbitals approach to designate the base-pairs' HOMO and LUMO. Namely, the basepair HOMO/LUMO $(H / L)$ wavefunction reads

$$
\Psi_{H / L}^{b p}(\mathbf{r})=\mathcal{C}_{1} \Psi_{H / L}^{b(1)}(\mathbf{r})+\mathcal{C}_{2} \Psi_{H / L}^{b(2)}(\mathbf{r}),
$$

where $\Psi_{H / L}^{b(1)}(\mathbf{r}), \quad \Psi_{H / L}^{b(2)}(\mathbf{r}) \quad$ are the corresponding HOMO/LUMO orbitals of the two bases (1) and
(2) forming the base-pair. Inserting Eq. (3) into Schrödinger equation $H^{b p} \Psi_{H / L}^{b p}(\mathbf{r})=E_{H / L}^{b p} \Psi_{H / L}^{b p}(\mathbf{r})$ (where $E_{H / L}^{b p}$ denotes the HOMO/LUMO basepair energy), multiplying once with $\Psi_{H / L}^{b(1) \star}(\mathbf{r})$ and once with $\Psi_{H / L}^{b(2) \star}(\mathbf{r})$, and integrating over all space, using $\Psi_{H / L}^{b(1)}(\mathbf{r})=\sum_{i=1}^{N_{1}} c_{i(1)}^{H / L} p_{z}^{i(1)}(\mathbf{r})$,

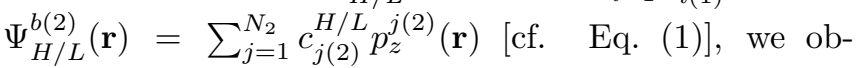
tain the following system of equations

$$
\begin{aligned}
E_{H / L}^{b(1)} \mathcal{C}_{1}+t_{H / L} \mathcal{C}_{2} & =E_{H / L}^{b p} \mathcal{C}_{1} \\
t_{H / L}^{\star} \mathcal{C}_{1}+E_{H / L}^{b(2)} \mathcal{C}_{2} & =E_{H / L}^{b p} \mathcal{C}_{2}
\end{aligned}
$$

Here we have assumed that $p_{z}$ orbitals belonging to atoms of different bases are orthogonal and also that $\int d^{3} r \Psi_{H / L}^{b(m) \star} H^{b p} \Psi_{H / L}^{b(m)} \approx \int d^{3} r \Psi_{H / L}^{b(m) \star} H^{b} \Psi_{H / L}^{b(m)}=$ $E_{H / L}^{b(m)}$, for $m=1$ or 2 . The overlap integral, $t_{H / L}=$ $\int d^{3} r \Psi_{H / L}^{b(1) \star} H^{b p} \Psi_{H / L}^{b(2)}$, which expresses the hopping parameter for a charge (hole/electron) transfer between the two bases of the base-pair, equals

$$
t_{H / L}=\sum_{i=1}^{N_{1}} \sum_{j=1}^{N_{2}} c_{i(1)}^{H / L \star} c_{j(2)}^{H / L} V_{i j},
$$

where $V_{i j}=\int d^{3} r p_{z}^{i(1) \star}(\mathbf{r}) H^{b p} p_{z}^{j(2)}(\mathbf{r})$.

Because the base wavefunctions $\Psi^{b}(\mathbf{r})$ are real, the coefficients $c_{i}$ in Eq. (11) are real, and the same holds for the overlap integrals $t_{H / L}$, i.e., $t_{H / L}^{\star}=t_{H / L}$. The matrix elements $V_{i j}$ are generally provided through the SlaterKoster expression [41]:

$$
V_{i j}=V_{p p \sigma} \sin ^{2} \phi+V_{p p \pi} \cos ^{2} \phi,
$$

where $\phi$ denotes the angle formed by the line connecting atoms $i$ and $j$ and the plane perpendicular to $p_{z}$ orbitals (i.e. the plane of bases). More details regarding the meaning of $V_{p p \sigma}$ and $V_{p p \pi}$ matrix elements can be found in Ref. [40].

For atoms belonging to different bases within a basepair, i.e. the case of interest in this subsection, the angle $\phi=0$ and $V_{i j}=V_{p p \pi}$. Note that for intra-base covalently bonded neighboring atoms, $V_{p p \pi}$ are the interatomic matrix elements proposed by Harrison [40], cf. Eq. (2). However, Harrison's relations are valid for interatomic distances of the order of those of covalent bonds. When dealing with larger interatomic distances, e.g. between atoms belonging to different molecules, the proportional to $1 / d^{2}$ expressions of Harrison [40] are replaced by appropriate exponentially decaying expressions of the form [42, 43]

$$
V_{p p \pi}=A e^{-\beta\left(d-d_{0}\right)},
$$

where the constants $A, \beta$ are determined through the requirement that at a typical covalent bond distance, $d_{0}$, 
the values of this expression and its derivative in respect to $d$, coincide with those of Harrison's expressions given in Eq. (2). This yields $A=-0.63 \hbar^{2} / m d_{0}^{2}$ and $\beta=2 / d_{0}$. Here we choose $d_{0}=1.35 \AA$.

Using Eq.(7) for $V_{i j}$ (since $V_{i j}$ is equal to $V_{p p \pi}$ for atoms of different bases within the same base-pair, i.e. $\phi=0)$ and the known coefficients $c_{i}^{H / L}$ of the base HOMO/LUMO (obtained as described in the previous subsection), then $t_{H / L}$ are calculated through Eq. (5). The quantities $E_{H / L}^{b(1)}$ and $E_{H / L}^{b(2)}$ in Eq. (44) are the HOMO/LUMO eigenenergies of the corresponding bases. Therefore, the $2 \times 2$ system of Eq. (4) can be analytically solved to determine $\mathcal{C}_{1}, \mathcal{C}_{2}$, and $E_{H / L}^{b p}$. To obtain the HOMO (LUMO) of the base-pair, the higher (lower) energy solution of the $2 \times 2$ system (4) is considered and $E_{H}^{b p}$ $\left(E_{L}^{b p}\right)$ is the corresponding eigenenergy. From the values $\mathcal{C}_{1}$ and $\mathcal{C}_{2}$ of the solution, the base-pair HOMO/LUMO wavefunction is obtained through Eq. (3). For later use it is mentioned that by multiplying $\mathcal{C}_{1}$ and $\mathcal{C}_{2}$ with the LCAO coefficients of the corresponding base wavefunctions $\Psi_{H / L}^{b(1)}$ and $\Psi_{H / L}^{b(2)}$ [cf. Eq. (1)], the base-pair wavefunction (3) can be equivalently written as

$$
\Psi_{H / L}^{b p}(\mathbf{r})=\sum_{i=1}^{N} C_{i}^{H / L} p_{z}^{i}(\mathbf{r})
$$

Here the sum is extended over the $N$ atoms (contributing $p_{z}$ electrons in $\pi$ bonds) of the whole base-pair $(N=18$ for A-T, while $N=19$ for G-C).

\section{Tight-binding parameters for charge transfer in B-DNA}

HOMO and LUMO energies of bases (or base-pairs) as well as hopping parameters between successive bases (or base-pairs) -calculated through a similar procedure like in the previous subsection, cf. Eq. (5)- provide an estimate of the parameters used in tight-binding models that describe charge transport along DNA. Such phenomenological models facilitate larger scale simulations, reaching a mesoscopic level of description. Depending on the particular problem of interest, a tight-binding description at the base-pair level, or at the single-base level, may be more appropriate. The relevant parameters for both cases are discussed below and their estimated values are presented in Section III.

\section{Description at the base-pair level}

A tight-binding description of charge transfer between successive base-pairs $\ldots, \lambda-1, \lambda, \lambda+1, \ldots$ of doublestranded DNA can be obtained considering that extra electrons inserted in DNA travel through LUMOs, while inserted holes travel through HOMOs. In this approximation the time-dependent single carrier (hole/electron) wavefunction of the whole macromolecule, $\Psi_{H / L}^{D N A}(\mathbf{r}, t)$, is considered as a linear combination of base-pair wavefunctions with time-dependent coefficients, i.e.,

$$
\Psi_{H / L}^{D N A}(\mathbf{r}, t)=\sum_{\lambda} A_{\lambda}(t) \Psi_{H / L}^{b p(\lambda)}(\mathbf{r})
$$

where $\Psi_{H / L}^{b p(\lambda)}(\mathbf{r})$ is the $\lambda^{\text {th }}$ base-pair's HOMO/LUMO wavefunction and the sum is extended over all base-pairs of the DNA molecule under consideration.

Starting from the time-dependent Schrödinger equation, $i \hbar \frac{d \Psi_{H / L}^{D N A}}{d t}=H^{D N A} \Psi_{H / L}^{D N A}$, and following a similar procedure and assumptions similar to the ones of the previous subsection, one obtains that the time evolution of the coefficients $A_{\lambda}(t)$ obeys the tight-binding equations

$$
i \hbar \frac{d A_{\lambda}}{d t}=E_{H / L}^{b p(\lambda)} A_{\lambda}+t_{H / L}^{b p(\lambda ; \lambda-1)} A_{\lambda-1}+t_{H / L}^{b p(\lambda ; \lambda+1)} A_{\lambda+1} .
$$

Here, $E_{H / L}^{b p(\lambda)}$ is the HOMO/LUMO energy of base-pair $\lambda$, as obtained in the previous subsection IIB. Depending on the actual sequence of the DNA molecule under consideration, two values are possible for the on-site energies $E_{H / L}^{b p(\lambda)}$ of Eq. (10), corresponding to A-T or G-C base-pairs. Using the HOMO or LUMO base-pair wavefunctions $\left(\Psi_{H}^{b p}\right.$ or $\left.\Psi_{L}^{b p}\right)$, cf. Eq. (8), the corresponding hopping parameters $\left(t_{H}^{b p}\right.$ or $\left.t_{L}^{b p}\right)$ between successive basepairs are obtained by

$$
t_{H / L}^{b p\left(\lambda ; \lambda^{\prime}\right)}=\sum_{i=1}^{N_{\lambda}} \sum_{j=1}^{N_{\lambda^{\prime}}} C_{i(\lambda)}^{H / L \star} C_{j\left(\lambda^{\prime}\right)}^{H / L} V_{i j},
$$

where the indices $\lambda, \lambda^{\prime}$ denote neighboring base-pairs and $V_{i j}=\int d^{3} r p_{z}^{i(\lambda) \star}(\mathbf{r}) H^{D N A} p_{z}^{j\left(\lambda^{\prime}\right)}(\mathbf{r})$. The sums over $i$ and $j$ in Eq. (11) extend up to the total number of atoms $N_{\lambda}$ and $N_{\lambda^{\prime}}$, respectively, constituting the corresponding base-pair [cf. Eq. (8)]. Here there is a difference with the hopping integrals in Eq. (5), since in the latter case the sums up to $N_{1}$ and $N_{2}$ extend over the total number of atoms constituting the corresponding bases. The matrix elements $V_{i j}$ in Eq. (11) are given by the semi-empirical Slater-Koster expression, Eq. (6). Now $\phi \neq 0$ and $V_{p p \pi}$ is obtained through Eq. (7), as before. $V_{p p \sigma}$ is also obtained from the same expression, Eq. (7) but with a different preexponential coefficient, $A=2.22 \hbar^{2} / m d_{0}^{2}$, resulting from the constant 2.22 which appears in the corresponding to Eq. (2) Harrison's formula for $V_{p p \sigma}$ [40]. Therefore, using standard expressions from solid state physics [40, 41, 42, 43], we are able to calculate the interatomic matrix elements $V_{i j}$. The Slater-Koster expression of Eq. (6) (see Table I of Ref. [41]) with matrix elements $V_{p p \pi}$ and $V_{p p \sigma}$ of the Harrison's type [40], is typically used in solid state physics and it has been also applied in the case of DNA charge transfer in Ref. [4]. Our methodology for calculating the overlap matrix elements is very similar to that of the latter work. The difference is that 
we use the exponentially decaying expressions of the form of Eq. (77) for $V_{p p \pi}$ and $V_{p p \sigma}$, adopted from Refs. [42, 43], instead of the fitting of similar expressions with $a b$ initio calculations as used in Ref. [4]. All coefficients and matrix elements in Eq. (11) are real, resulting in real $t_{H / L}^{b p\left(\lambda ; \lambda^{\prime}\right)}=t_{H / L}^{b p\left(\lambda^{\prime} ; \lambda\right)}$.

Knowledge of the coefficients $C_{i}^{H / L}$ from the base-pair wavefunctions of Sec. ПB [cf. Eq. (8)] and of the matrix elements $V_{i j}$ from the geometrical structure of DNA and the formulae discussed above, allows the calculation of the hopping integrals $t_{H / L}^{b p\left(\lambda ; \lambda^{\prime}\right)}$ [cf. Eq. (11)] which appear as parameters in the tight-binding Eq. (10). Therefore, one can use the tight-binding parameters $E_{H / L}^{b p(\lambda)}$ and $t_{H / L}^{b p\left(\lambda ; \lambda^{\prime}\right)}$ computed in this work (cf. Table【and Table III respectively, in Sec. III), in order to numerically solve the system of equations (10) and obtain, through $A_{\lambda}(t)$, the time evolution of a charge [cf. Eq. (9)] propagating along any DNA segment.

\section{Description at the single-base level}

The tight-binding description at the single-base level is similar to that in the previous subsection. The differ- ence is that the carrier wavefunction of the whole macromolecule is now considered as a linear combination of single base wavefunctions, instead of base-pair wavefunctions. Therefore

$$
\Psi_{H / L}^{D N A}(\mathbf{r}, t)=\sum_{\lambda}\left[A_{\lambda}(t) \Psi_{H / L}^{b(\lambda, 1)}(\mathbf{r})+B_{\lambda}(t) \Psi_{H / L}^{b(\lambda, 2)}(\mathbf{r})\right]
$$

where $\lambda$ denotes base-pairs, the sum is again over all successive base-pairs of DNA, and $\Psi_{H / L}^{b(\lambda, 1)}, \Psi_{H / L}^{b(\lambda, 2)}$ are HOMO/LUMO wavefunctions of bases in the $\lambda^{\text {th }}$ basepair, located at the one and the other DNA strands, respectively.

Under the same assumptions as previously and considering only neighboring hoppings (between adjacent basepairs), the tight-binding equations for the time dependent coefficients of Eq. (12) read

$$
\begin{aligned}
& i \hbar \frac{d A_{\lambda}}{d t}=E_{H / L}^{b(\lambda, 1)} A_{\lambda}+t_{H / L}^{b(\lambda, 1 ; \lambda, 2)} B_{\lambda}+t_{H / L}^{b(\lambda, 1 ; \lambda-1,1)} A_{\lambda-1}+t_{H / L}^{b(\lambda, 1 ; \lambda+1,1)} A_{\lambda+1}+t_{H / L}^{b(\lambda, 1 ; \lambda-1,2)} B_{\lambda-1}+t_{H / L}^{b(\lambda, 1 ; \lambda+1,2)} B_{\lambda+1} \\
& i \hbar \frac{d B_{\lambda}}{d t}=E_{H / L}^{b(\lambda, 2)} B_{\lambda}+t_{H / L}^{b(\lambda, 2 ; \lambda, 1)} A_{\lambda}+t_{H / L}^{b(\lambda, 2 ; \lambda-1,2)} B_{\lambda-1}+t_{H / L}^{b(\lambda, 2 ; \lambda+1,2)} B_{\lambda+1}+t_{H / L}^{b(\lambda, 2 ; \lambda-1,1)} A_{\lambda-1}+t_{H / L}^{b(\lambda, 2 ; \lambda+1,1)} A_{\lambda+(13)}
\end{aligned}
$$

Here $E_{H / L}^{b}$ are base HOMO/LUMO energies and the hopping parameters $t_{H / L}^{b}$ are interbase transfer integrals of the general form of Eq. (5) with $V_{i j}$ given by Eq. (6). $\phi=0$ for interbase transfer integrals within the same base-pair (interstrand intra-base-pair hoppings), $t_{H / L}^{b(\lambda, 1 ; \lambda, 2)}=t_{H / L}^{b(\lambda, 2 ; \lambda, 1)}$, while $\phi \neq 0$ for interbase transfer integrals between successive bases within the same strand (intrastrand hoppings), $t_{H / L}^{b(\lambda, i ; \lambda \pm 1, i)}$, or between diagonally located, at different strands, bases (interstrand inter-base-pair hoppings), $t_{H / L}^{b(\lambda, i ; \lambda \pm 1, j)}$ with $i \neq j$. $V_{p p \pi}$ and $V_{p p \sigma}$ are given through exponentially decaying Eq. (7), as discussed before.

A tight-binding description at the single-base level requires solving of a double number of differential equations, Eq. (13), compared to the description at the basepair level, Eq. (10). However, it allows individual base properties to be taken into account when necessary. Section III contains the tight-binding parameters which appear in Eq. (13); see Table I for $E_{H / L}^{b}$, Table IV for
$t_{H / L}^{b(\lambda, i ; \lambda, j)}$, Table V for $t_{H / L}^{b(\lambda, i ; \lambda \pm 1, i)}$, and Tables VI and VII for $t_{H / L}^{b(\lambda, i ; \lambda \pm 1, j)}$ with $i \neq j$.

\section{RESULTS AND DISCUSSION}

The results and their discussion have been organized in two subsections. In subsection III A we present our calculations for the HOMO and LUMO energies as well as for the corresponding wavefunctions of the four DNA bases, adenine, guanine, cytosine, and thymine, and of the two base-pairs, adenine-thymine and guanine-cytosine. We compare our results with available experimental data and previous calculations using methods from first principles.

Subsection IIIB contains our calculations for the charge transfer hopping parameters between successive base-pairs (Sec. IIIB 1) or between neighboring DNA bases (Sec. III B 2), and provides estimates for the corresponding tight-binding parameters described previously in Section II C. We present results for both electrons and holes and for all possible combinations of successive bases 


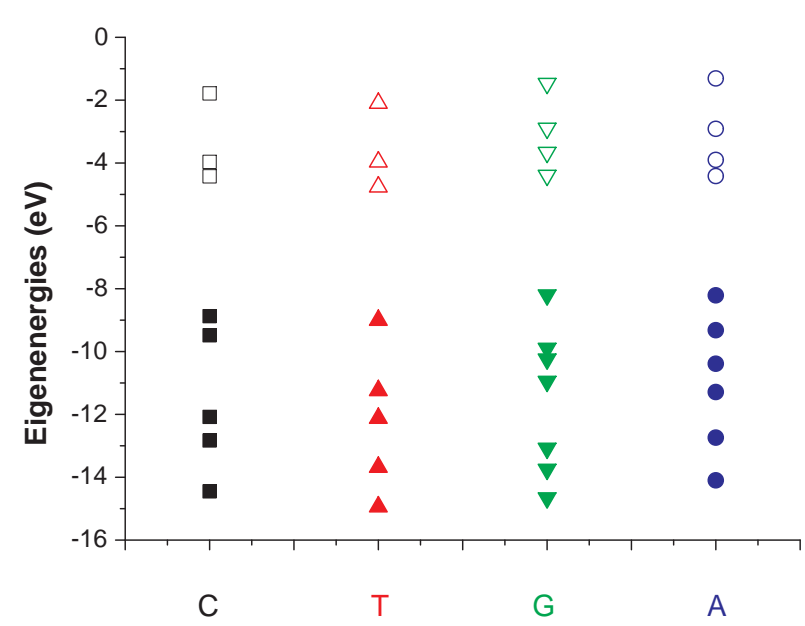

TABLE I: For each DNA base (first row) are shown: the number of atoms participating in $\pi$ bonds (second row), the total number of contributed $p_{z}$ electrons (third row), the $\pi \mathrm{HOMO}$ energy $E_{H}^{b(i s o l .)}$ (fourth row) and the $\pi$ LUMO energy $E_{L}^{b \text { (isol.) }}$ (fifth row) of the isolated base, as well as the energy of the first $\pi-\pi^{*}$ transition $E_{\pi-\pi^{*}}=E_{L}^{b(i s o l .)}-E_{H}^{b(\text { isol.) }}$ (sixth row), obtained in this work. We also show experimental values of the ionization energy $I E$ (seventh row) and the first $\pi-\pi^{*}$ transition (eighth row), as well as existing theoretical predictions using methods from first principles for $I E$ (ninth row) and $E_{\pi-\pi^{*}}$ (tenth row). Finally, we list the $\pi \mathrm{HOMO}$ and $\pi$ LUMO energies, $E_{H}^{b}$ (eleventh row) and $E_{L}^{b}$ (twelfth row), respectively, of the bases when they are distorted within the base-pairs of B-DNA (see text), as obtained in our work. The quantities $E_{H}^{b}$ in eleventh row and $E_{L}^{b}$ in twelfth row represent the parameters $E_{H / L}^{b(\lambda, i)}$ which appear in Eq. (13). All energies are given in $\mathrm{eV}$. FIG. 1: (Color online) $\pi$ molecular structure of the
bases: cytosine $(\mathrm{C})$, thymine $(\mathrm{T})$, guanine $(\mathrm{G})$, and
(A). Full (empty) symbols correspond to occupied
pied) molecular orbitals.

or base-pairs.

\section{DNA base} \begin{tabular}{|l|l|l|l|}
\hline Adenine & Thymine & Guanine & Cytosine \\
\hline
\end{tabular}

\begin{tabular}{c|c|c|c}
\hline \hline 10 & 8 & 11 & 8 \\
\hline 12 & 10 & 14 & 10 \\
\hline-8.2 & -9.0 & -8.2 & -8.9 \\
\hline-4.4 & -4.8 & -4.4 & -4.4 \\
\hline 3.8 & 4.2 & 3.8 & 4.5 \\
\hline & & & \\
$8.4-8.5$ & $9.0-9.2$ & $8.2-8.3$ & 8.9 \\
\hline & & & \\
$4.5-4.8$ & $4.6-4.7$ & $4.3-4.5$ & $4.5-4.7$ \\
\hline & & & \\
$8.2-8.6$ & $9.1-9.7$ & $7.8-8.3$ & $8.9-9.4$ \\
\hline & & & \\
$4.5-5.3$ & $4.9-5.3$ & $4.4-5.3$ & $4.2-4.7$ \\
\hline-8.3 & -9.0 & -8.0 & -8.8 \\
\hline-4.4 & -4.9 & -4.5 & -4.3 \\
\hline
\end{tabular}

\section{A. HOMO and LUMO energies and wavefu}

atoms in $\pi$ bonds

\begin{tabular}{l|l|l|l} 
& Adenine & Thymine & Guan \\
\hline
\end{tabular}

Paxdelefictens

$E_{L}^{b(\text { isol. })}$

$E_{\pi-\pi^{*}}$

$I E^{\mathrm{exp}}$

$[45,46,47,48,49]$ $E_{\pi-\pi^{*}}^{\exp }$

$[50,51,52,53,54,55,56,57,58,59,60,61,62]$

Before starting our discussion about the HO $[63,64,65]$ $I E^{\text {nIst pr. }}$
$[63,64,65]$ LUMO of DNA bases, we show in Fig. 1 the $\frac{\pi^{\pi} \text { etêc- }}{4}$ tronic structure of all bases, as obtained using th $\left[66_{6} 67068,69,70,71,72,73,74,75,76,77\right]$

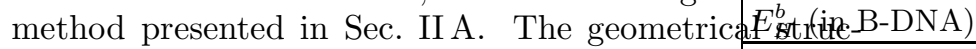
ture of bases and the corresponding interatomic diEtar(ineB-DNA) have been obtained from Ref. 44]. Occupied orbitals are shown by full symbols in Fig. 11 while unoccupied ones by empty symbols. The second and third row of Table Ilist -for each base of the corresponding column- the number of atoms participating in $\pi$-bonds and the total number of $p_{z}$ electrons in the molecule, respectively. For example, adenine has twelve $p_{z}$ electrons, which occupy, by pairs of opposite spin, the six lowest energy levels of A shown in Fig. 1 .

Table [1 summarizes our results for the HOMO and LUMO energies of DNA bases. Relevant experimental values for the vertical ionization energy (which is equal to the absolute value of HOMO energy) and the first $\pi-\pi^{*}$ transition (i.e. the energy difference between LUMO and HOMO) are also shown, along with corresponding results of previous theoretical calculations using methods from first principles. When the bases form base-pairs within DNA, they are slightly deformed in comparison to their structure when isolated. The HOMO and LUMO energies of the distorted bases in B-DNA (shown in the last two rows of Table (I) may differ from those of the isolated bases, and these values provide the relevant on-site TABLE II: For the two B-DNA base-pairs (first row) are shown: the $\pi \mathrm{HOMO}$ energy $E_{H}^{b p}$ (second row), the $\pi \mathrm{LUMO}$ energy $E_{L}^{b p}$ (third row), and the corresponding first $\pi-\pi^{*}$ transition energy $E_{\pi-\pi^{*}}=E_{L}^{b p}-E_{H}^{b p}$ (fourth row), as obtained in this work. We also list existing theoretical predictions using methods from first principles, for the HOMO energy (fifth row) and the first $\pi-\pi^{*}$ transition (sixth row). The quantities $E_{H}^{b p}$ in the second row and $E_{L}^{b p}$ in the third row represent the parameters $E_{H / L}^{b p(\lambda)}$ which appear in Eq. (10). All energies are given in $\mathrm{eV}$.

\begin{tabular}{|l|c|c|}
\hline B-DNA base-pair & A-T & G-C \\
\hline \hline$E_{H}^{b p}$ & -8.3 & -8.0 \\
\hline$E_{L}^{b p}$ & -4.9 & -4.5 \\
\hline$E_{\pi-\pi^{*}}$ & 3.4 & 3.5 \\
\hline$E_{H}^{b p \text { first pr. }[64,65,71,78,79]}$ & $-(7.8-8.2)$ & $-(6.3-7.7)$ \\
\hline$E_{\pi-\pi^{*}}^{\text {first pr. }}[76,79]$ & 6.4 & $4.3-6.3$ \\
\hline
\end{tabular}
energies $E_{H / L}^{b}$ in the tight-binding Eq. (13). 

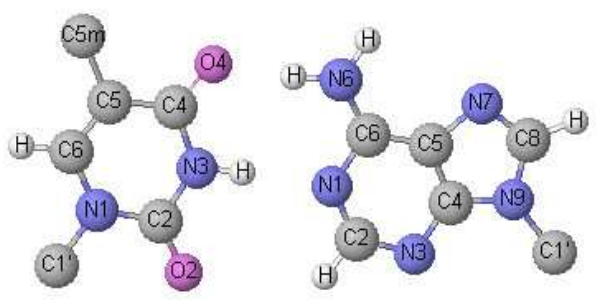

Jmol
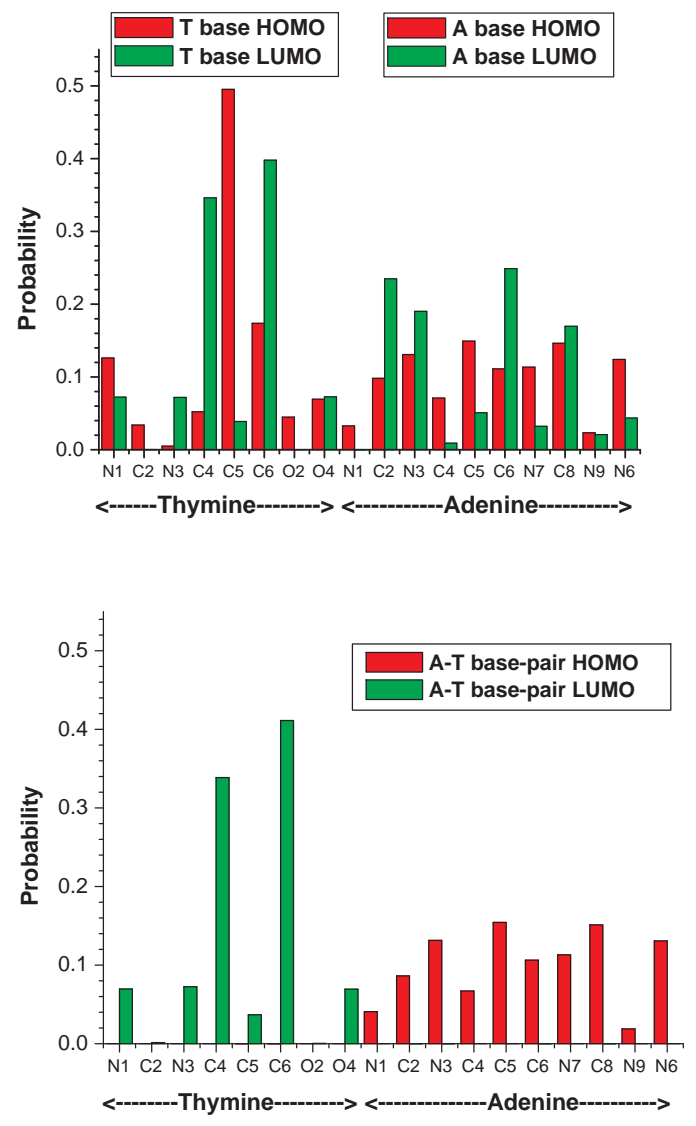

FIG. 2: (Color online) Top: geometrical structure of adenine (right) and thymine (left), within an A-T base-pair. It is shown the standard numbering of atoms, as also used in this work. $\mathrm{C}^{\prime}$ denote deoxyribose carbons and C5m thymine's methyl carbon, not participating in $\pi$ bonding. Middle: atomic occupation probabilities, $\left|c_{i}\right|^{2}$ [cf. Eq. (1)], for the HOMO and LUMO wavefunctions of isolated adenine (right) and thymine (left) bases. Bottom: atomic occupation probabilities, $\left|C_{i}\right|^{2}$ [cf. Eq. (8)], for the HOMO and LUMO wavefunctions of A-T base-pair.

In Table we report results for the HOMO and LUMO energies (and the HOMO-LUMO energy gap) of the two B-DNA base-pairs, using the procedure desribed in Section IB. Corresponding values obtained previously from first principles methods are also shown.

Figure 2 (Figure 3) presents for A, T, and A-T (for

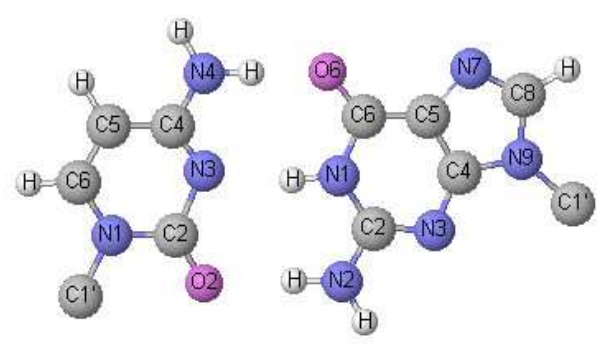

$\mathrm{Jmol}$
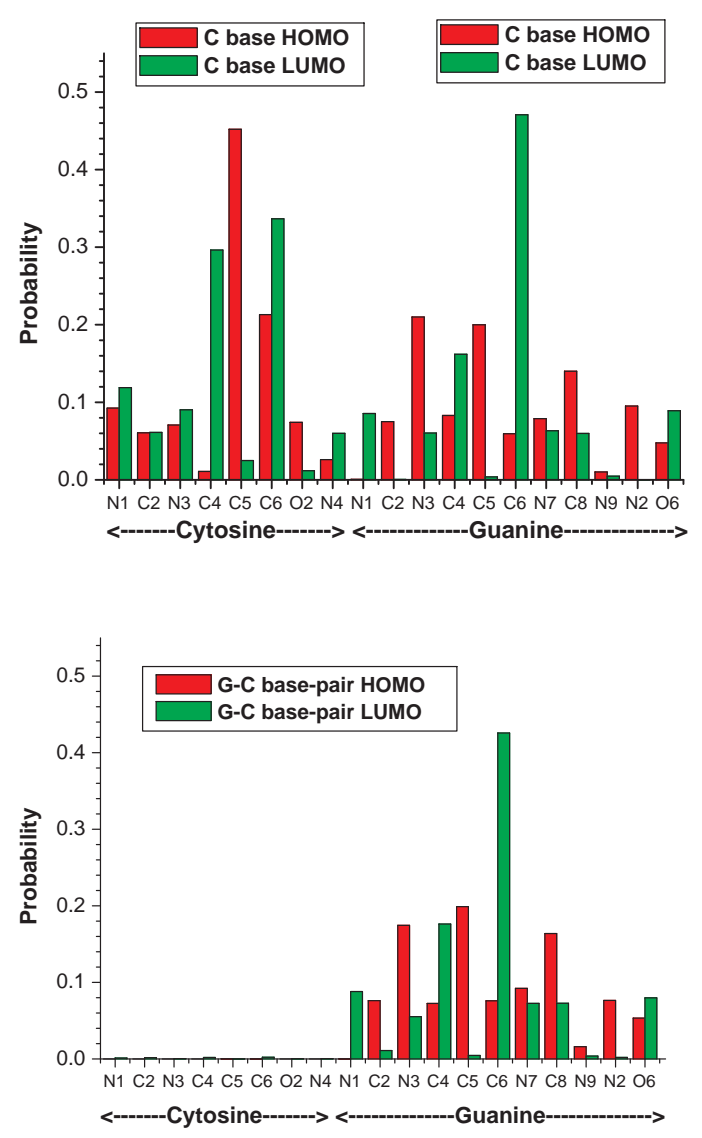

FIG. 3: (Color online) Top: geometrical structure of guanine (right) and cytosine (left), within a G-C base-pair. It is shown the standard numbering of atoms, as also used in this work. $\mathrm{C} 1^{\prime}$ denote deoxyribose carbons, not participating in $\pi$ bonding. Middle: atomic occupation probabilities, $\left|c_{i}\right|^{2}[\mathrm{cf}$. Eq. (1)], for the HOMO and LUMO wavefunctions of isolated guanine (right) and cytosine (left) bases. Bottom: atomic occupation probabilities, $\left|C_{i}\right|^{2}$ [cf. Eq. (8)], for the HOMO and LUMO wavefunctions of G-C base-pair.

G, C, and G-C) the corresponding intramolecular occupation probabilities of holes or electrons, obtained from the calculated HOMO or LUMO wavefunctions, respectively. This is shown through the squared coefficients $\left|c_{i}\right|^{2}$ (or $\left|C_{i}\right|^{2}$ for base-pairs) in the expansion of Eq. (1) (or Eq. (8) for base-pairs) of the corresponding wavefunc- 
tion. These squared coefficients represent the probabilities of finding an electron or hole (LUMO or HOMO, respectively) located at the corresponding atom of the base or the base-pair. The structures of the bases, prepared with the open-source software for chemical structures Jmol [80], are shown on the top of each figure. The standard numbering of atoms has been used.

A detailed discussion regarding the HOMO and LUMO wavefunctions is carried out below, separately for each isolated base and for the two B-DNA base-pairs.

\section{Adenine}

Our calculations show that the HOMO energy of adenine is $-8.2 \mathrm{eV}$. This slightly underestimates the experimental value of the ionization energy $(8.4 \mathrm{eV}$ [45], $8.5 \mathrm{eV}$ [48, 49]) by $0.2-0.3 \mathrm{eV}$. Our result is similar to that of Ref. 64], where using ab initio calculations the HOMO energy was found $-8.24 \mathrm{eV}$. Other $a b$ initio calculations predicted the ionization energy between 8.3-8.6 eV 63 . In good accordance with the measured values is also the $a b$ initio prediction of $8.5 \mathrm{eV}$ in Ref. [65].

The calculated HOMO wavefunction (see Fig. 2 middle right) is distributed all over adenine. In descending order, it has its main amplitude in atoms C5, C8, N3, N6, N7, C6, C2, C4, while the lowest probabilities exist in atoms N1 and N9. These results agree with those from Ref. [21], since in both cases the HOMO wavefunction is distributed all over adenine and the lowest probabilities are found at the same atoms. Our results are also in agreement with the outcome of density functional theory calculations [81], where the $\pi$ HOMO wavefunction was found to be delocalized along adenine, with greater probabilities in atoms $\mathrm{N} 7, \mathrm{~N} 3, \mathrm{C} 6, \mathrm{C} 5, \mathrm{C} 8$, and the lowest in atom $\mathrm{N} 9$, as well as with recent time-dependent density functional theory predictions [76], where the HOMO wavefunction is distributed all over the molecule except from atom N9.

We find the LUMO energy of adenine at $-4.4 \mathrm{eV}$. According to our calculations, the first $\pi-\pi^{*}$ transition energy is $E_{\pi-\pi^{*}}=3.8 \mathrm{eV}$. The deviation from the experimental value $(4.5-4.8 \mathrm{eV}[50,53,54,56,57,61,62])$ is $0.7-1.0 \mathrm{eV}$. The $E_{\pi-\pi^{*}}$ value reported from several first principles methods is around 4.5-5.3 eV 68, 70, 72, 75, 76, 77], i.e. the corresponding deviation from the experimental observations is $0-0.8 \mathrm{eV}$. It must be mentioned here that, although it is generally accepted for all DNA bases that the HOMO-LUMO energy gap corresponds to the first $\pi-\pi^{*}$ transition [45, 48, 49, 54, 57], in Ref. 77] the HOMO-LUMO gap of adenine is attributed to a $n$ $\pi^{*}$ transition, while in Refs. [75, 76] the $\pi-\pi^{*}$ and $n-\pi^{*}$ transitions are predicted to be very close.

Regarding the LUMO wavefunction of adenine, it can be seen from Fig. 2 (middle right) that the highest probabilities are found in atoms $\mathrm{C} 6, \mathrm{C} 2, \mathrm{~N} 3$, and $\mathrm{C} 8$, while the remaining atoms are occupied by much smaller probabilities $(\lesssim 5 \%)$. Our findings agree with those of Ref. [21], where the LUMO wavefunction is localized in atoms C8, C6, C2, N3, and N7. The only difference from our findings is the existence of a relatively higher probability in atom N7. In both results the lowest probabilities appear in the same atoms. Our findings are also in good accordance with the results of Ref. [76], where the LUMO wavefunction is localized in the same atoms $(\mathrm{C} 8, \mathrm{C} 6, \mathrm{C} 2$, N3).

\section{Thymine}

Thymine's HOMO energy is found $-9.0 \mathrm{eV}$, which is in agreement with the experimental value of ionization energy, $I E_{\mathrm{T}}=9.0-9.2 \mathrm{eV}$ [45, 46, 47, 49]. The ab initio predictions of Refs. 64 and 65] fall also within the range of experimental results, as they found the HOMO energy at $-9.14 \mathrm{eV}$ and $-9.16 \mathrm{eV}$, respectively. However, other ab initio calculations [63] give the ionization energy between 9.4-9.7 eV, which is in worse agreement with the experimental data (a deviation of $0.2-0.7 \mathrm{eV}$ ).

According to our calculations the HOMO wavefunction of thymine (cf. Fig. 2, middle left) is mainly localized at the atom C5 (with almost 50\% probability) and at its nearby atoms $\mathrm{C} 6$ and N1 (with total probability around $30 \%$ ). The remaining atoms have probabilities less than $10 \%$ each, with a negligible probability at the atom N3.

We find that the LUMO energy of thymine is $-4.8 \mathrm{eV}$. Looking at the first $\pi-\pi^{*}$ transition energy, our prediction of $4.2 \mathrm{eV}$ underestimates the experimental results (4.6-4.7 eV [50, 51, 52, 53, 54, 56, 57]) by $0.4-0.5 \mathrm{eV}$. Calculated $E_{\pi-\pi^{*}}$ values from several ab initio methods are between 4.9-5.3 eV [67, 69, 75, 77], which overestimate the observed values by $0.2-0.7 \mathrm{eV}$. We mention that Refs. [67, 69, 75, 77] attribute the HOMO-LUMO gap of thymine to a $n-\pi^{*}$ transition.

Fig. 2 (middle left) shows that the LUMO wavefunction of thymine exhibits its main amplitudes in atoms C6 and $\mathrm{C} 4$, with a total probability around $75 \%$. All other atoms have less than $10 \%$ probability each, with vanishing amplitudes at the atoms $\mathrm{C} 2$ and $\mathrm{O} 2$. Our predictions agree with those from Ref. 76], where the LUMO wavefunction is mainly localized in atoms $\mathrm{C} 4, \mathrm{C} 5$, and $\mathrm{C} 6$.

\section{Guanine}

The HOMO energy of guanine is $-8.2 \mathrm{eV}$ according to our results. Our prediction for its ionization energy almost coincides with the experimental observations (8.24 $\mathrm{eV}$ [45] and $8.28 \mathrm{eV}$ [48]). Previous ab initio calculations have found the ionization energy of guanine at $8-8.3 \mathrm{eV}$ [63], $7.75 \mathrm{eV}$ [64], and $8.21 \mathrm{eV}$ [65]. These values deviate from the experimental ones by $0-0.5 \mathrm{eV}$.

We observe in Fig. 3 (middle right) that the HOMO wavefunction of guanine is extended over a large part of the molecule, containing the atoms N2-C2-N3-C4-C5-N7C8 (with total probability around 90\%). The atoms N3 
and C5 present the highest probabilities (around 20\% each), while about $15 \%$ probability exists in atom $\mathrm{C} 8$. A vanishing probability appears at the atom N1 and a very small one at N9. Our results agree with those of Ref. [81], where the main amplitude appears in the part N2-C2-N3-C4-C5 of the molecule and a lower probability also exists in atom $\mathrm{C} 8$. The main difference between the two calculations is the existence of a probability $18 \%$ at the atom $\mathrm{O} 6$ in Ref. 81], compared to $5 \%$ in our case. Similar to our findings are the predictions obtained from the $a b$ initio method of Ref. [82], where the HOMO wavefunction is mainly localized at the same part of guanine, while there is also agreement with the results of Ref. [76], where the HOMO is predicted to be distributed all over the molecule apart from the atoms N1, C6, and N9.

We find that Guanine's LUMO energy is $-4.4 \mathrm{eV}$. Regarding the first $\pi-\pi^{*}$ transition, our prediction is 3.8 $\mathrm{eV}$, which underestimates the observed values (between $4.3-4.5 \mathrm{eV}[50,53,54,59])$ by $0.5-0.7 \mathrm{eV}$. The first $\pi-\pi^{*}$ excitation energy obtained from several first principles methods is between $4.4-5.3 \mathrm{eV}$ 68, 75, 76, 77], presenting deviations of $0-0.8 \mathrm{eV}$ from the experimental observations.

Looking at the LUMO wavefunction of guanine (Fig. 3, middle right), we see that the main amplitude appears in atom $\mathrm{C} 6$ with $\approx 45 \%$ occupation probability. A significant probability also exists in atom C4 (more than 15\%). All other atoms have probabilities less than $10 \%$, with negligible amplitudes at $\mathrm{C} 2, \mathrm{~N} 2, \mathrm{C} 5$, and $\mathrm{N} 9$. These results differ from those of Ref. [82], since there the LUMO wavefunction exhibits higher probabilities at the atoms $\mathrm{C} 2, \mathrm{C} 4, \mathrm{~N} 1, \mathrm{C} 5$, and N9, in descending order. On the contrary, the present findings are in reasonable agreement with those of Ref. [76.

\section{Cytosine}

The HOMO energy of cytosine is found $-8.9 \mathrm{eV}$. Our prediction for the ionization energy coincides with the measured value of $8.94 \mathrm{eV}$ [45]. Corresponding values calculated from ab initio methods are $8.87 \mathrm{eV}$ [64], 8.88 $\mathrm{eV}$ [65], and 9.0-9.4 eV [63]. The former predictions estimate the experimental ionization energy equally well with our simple LCAO calculations, while the latter ones worse.

It can be seen from Fig. 3 (middle left) that the HOMO wavefunction of cytosine is mainly localized at C5 (occupation probability $\approx 45 \%$ ), presenting also a significant amplitude at the neighboring atom C6 (probability larger than 20\%). Atoms N1, C2, O2, and N3 exhibit probabilities between $5 \%-10 \%$, while $\mathrm{C} 4$ and $\mathrm{N} 4$ have the smaller probabilities (below $2 \%$ each). In Ref. [81] it was found that the atoms C5 and C6 accumulate only 25\% probability, while the higher amplitudes appear in atoms $\mathrm{O} 2$ and N3.

We find the LUMO energy of cytosine equal to -4.4 $\mathrm{eV}$. The first $\pi-\pi^{*}$ transition energy according to our re- sults $(4.5 \mathrm{eV})$ is compared well with the experimentally observed values $(4.5-4.7 \mathrm{eV}$ 50, 53, 54, 55, 57, 58, 59, 60, 77]). Results from first principles methods predict values between $4.2-4.7 \mathrm{eV}$, i.e. some of them are within the range of experimental values $[73,74,75$, while others are slightly below the observed values [66, 76].

Figure 3 (middle left) shows that the LUMO wavefunction of cytosine is mainly localized in atoms C6 and C4 with occupation probabilities around $30 \%$ each. A probability more than $10 \%$ appears in $\mathrm{N} 1$, while $\mathrm{C} 2, \mathrm{~N} 3$, and N4 have probabilities between $5 \%-10 \%$. These findings are in accordance with the results of Ref. 76], where the higher probabilities are found in atoms $\mathrm{C} 4, \mathrm{C} 5$, and $\mathrm{C} 6$.

\section{A-T base-pair}

As it has been already mentioned, the bases are slightly deformed within the base-pairs of B-DNA. This distorted form of the bases is appropriate when discussing basepair properties (HOMO and LUMO energies or wavefunctions), as opposed to the isolated form of the bases considered in the previous subsections. Therefore, before going to the HOMO and LUMO discussion of basepairs, we briefly mention the changes in bases' HOMO and LUMO due to the structural distortion within basepairs.

For adenine in the B-DNA conformation the HOMO energy is $E_{H}^{A}=-8.3 \mathrm{eV}$ (differing by $0.1 \mathrm{eV}$ in respect with the value of the isolated base, $-8.2 \mathrm{eV}$, see Table 【), while the LUMO energy remains unchanged $\left(E_{L}^{A}=-4.4\right.$ $\mathrm{eV}$ ). Regarding the HOMO and LUMO wavefunctions of adenine the results are almost similar in both conformations. For example in the case of the HOMO wavefunction, the highest difference of the occupation probabilities in respect with the results of the isolated base (Fig. 2. middle) is $-1.2 \%$ in atom $\mathrm{C} 2$, while in all other atoms the differences are below $1 \%$. In the case of LUMO wavefunction the differences are slightly higher, reaching $+4.1 \%$ in atom $\mathrm{C} 8,-3.3 \%$ in atom $\mathrm{C} 2,-2.4 \%$ in atom $\mathrm{N} 3$, and $+2.1 \%$ in atom $\mathrm{N} 7$, while in the other atoms the changes are below $1.2 \%$.

For thymine the HOMO energy using the B-DNA structure is invariable in respect with that of the isolated base $\left(E_{H}^{T}=-9.0 \mathrm{eV}\right)$, while the LUMO energy changes slightly from $-4.8 \mathrm{eV}$ in the isolated form to $E_{L}^{T}-4.9$ $\mathrm{eV}$ in the B-DNA conformation. The occupation probabilities for the HOMO (LUMO) wavefunction of thymine are almost similar in both cases and the differences are not larger than $0.5 \%(1.5 \%)$.

According to our method, the HOMO energy of A-T base-pair is $E_{H}^{A T}=-8.3 \mathrm{eV}$. Comparing with ab initio results, this value differs only by $0.1 \mathrm{eV}$ with the prediction of Ref. 64] $(-8.19 \mathrm{eV})$, it is very close to the ones calculated in Refs. 78] $(-8.12 \mathrm{eV})$ and [65] (-8.06 $\mathrm{eV}$ ), while there is a reasonable agreement with the value given in Ref. [71] $(-7.8 \mathrm{eV})$.

The LUMO energy of A-T base-pair is $E_{L}^{A T}=-4.9$ 
$\mathrm{eV}$. Therefore, the first $\pi-\pi^{*}$ excitation energy in our calculations is $3.4 \mathrm{eV}$. The prediction of $E_{\pi-\pi^{*}}$ obtained from first principles methods in Ref. [79] is $6.39 \mathrm{eV}$. It must be mentioned that according to the latter study the HOMO-LUMO gap is not assigned to a $\pi-\pi^{*}$ transition, but to a $\pi-\sigma^{*}$ transition [79].

Figure2(bottom) shows that for the A-T base-pair the HOMO wavefunction is completely localized in adenine, while, on the contrary, the LUMO is completely localized in thymine. This is justified because of the significantly higher HOMO energy of A with respect to that of $\mathrm{T}$ $\left(E_{H}^{A}-E_{H}^{T}=0.7 \mathrm{eV}\right)$ and the significantly lower LUMO energy of $\mathrm{T}$ with respect to that of $\mathrm{A}\left(E_{L}^{T}-E_{L}^{A}=-0.5\right.$ $\mathrm{eV})$, respectively, as compared with the corresponding overlap integrals of the bases' wavefunctions within the base-pair $\left(\left|t_{H}\right|=12 \mathrm{meV}\right.$ and $\left|t_{L}\right|=9 \mathrm{meV}$, see Table IV below). This also explains that $E_{H}^{A T}=E_{H}^{A}$ and $E_{L}^{A T}=E_{L}^{T}$, as well as that the electron (hole) distribution within the base-pair is identical with the corresponding distribution in thymine (adenine). The latter can be seen by a comparison of the corresponding wavefunctions in the middle and bottom panels of Fig. 2, taking also into account that adenine's HOMO and thymine's LUMO wavefunctions remain practically the same in the B-DNA and isolated conformations, as it is mentioned above.

\section{G-C base-pair}

The HOMO energy of guanine base in the base-pair conformation is $E_{H}^{G}=-8.0 \mathrm{eV}$. There is a difference of $0.2 \mathrm{eV}$ compared to the corresponding result of the isolated base $(-8.2 \mathrm{eV})$. Its LUMO energy in the B-DNA conformation $\left(E_{L}^{G}=-4.5 \mathrm{eV}\right)$ changes by $0.1 \mathrm{eV}$ in comparison to the value of $-4.4 \mathrm{eV}$ obtained for the isolated base. Concerning the HOMO wavefunction of guanine the highest differences of the occupation probabilities in respect with the results for the isolated base (shown in Fig. 3. middle) are $-3.5 \%$ in atom $\mathrm{N} 3$ and $+2.4 \%$ in atom C8. In all other atoms the differences are below $2 \%$. For the LUMO state the biggest difference appears in atom $\mathrm{C} 6$, which is $-4.2 \%$, while in the other atoms the differences do not exceed $1.6 \%$.

Regarding cytosine, the HOMO and LUMO energies change slightly (by $+0.1 \mathrm{eV}$ ), as compared to those of the isolated base. In particular, the predictions of cytosine's HOMO and LUMO energies within the base-pair conformation are $E_{H}^{C}=-8.8 \mathrm{eV}$ and $E_{L}^{C}=-4.3 \mathrm{eV}$, respectively. For the HOMO wavefunction, the differences of the occupation probabilities in the two base conformations are below $2.5 \%$ for all atoms. On the contrary, significant changes appear in the LUMO wavefunction of cytosine within the B-DNA conformation. The occupation probabilities differ by $+14.6 \%$ in atom $\mathrm{C} 2,-6.4 \%$ in atoms $\mathrm{C} 4$ and $\mathrm{C} 6,-4.1 \%$ in atom $\mathrm{N} 3$, and $+3.2 \%$ in atom N1, as compared to the isolated base LUMO, while in the other atoms the differences are below $2.5 \%$.

For the G-C base-pair, the obtained HOMO energy is
$E_{H}^{G C}=-8.0 \mathrm{eV}$. Several values calculated with methods from first principles have been reported in the literature: $-7.68 \mathrm{eV}$ 64], $-7.51 \mathrm{eV}$ [5], $-7.35 \mathrm{eV}$ 68], $-7.2 \mathrm{eV}$ 71], and $-6.27 \mathrm{eV}$ [79].

The calculated LUMO energy here is $E_{L}^{G C}=-4.5 \mathrm{eV}$. Concerning the first $\pi-\pi^{*}$ transition energy, our prediction is $3.5 \mathrm{eV}$. This result is in a reasonable accordance with the $E_{\pi-\pi^{*}}$ value obtained in Ref. [76] $(4.29 \mathrm{eV})$. However, these values differ from the one reported in Ref. 79] $(6.27 \mathrm{eV})$, where, similarly to the case of the A-T base-pair, the HOMO-LUMO gap is attributed to a $\pi-\sigma^{*}$ transition.

From Fig. 3 (bottom) we see that both HOMO and LUMO wavefunctions of a G-C base-pair are completely localized in guanine. The complete HOMO localization in guanine results again from the significantly higher HOMO energy of $\mathrm{G}$ in respect to that of $\mathrm{C}\left(E_{H}^{G}-E_{H}^{C}=\right.$ $0.8 \mathrm{eV})$, as compared with the hopping integral, $\left|t_{H}\right|=12$ $\mathrm{meV}$ in this case (see Table IV below). Similarly, for the LUMO wavefunction of G-C, its complete localization in guanine is due to the lower LUMO energy of $\mathrm{G}$ with respect to that of $\mathrm{C}\left(E_{L}^{G}-E_{L}^{C}=-0.2 \mathrm{eV}\right)$, as compared with the corresponding overlap integral of the bases' wavefunctions within the base-pair $\left(t_{L}=16 \mathrm{meV}\right.$, see Table IV]). These results are in accordance with our findings that $E_{H}^{G C}=E_{H}^{G}$ and $E_{L}^{G C}=E_{L}^{G}$. They also explain that the electron or hole distribution within the base-pair is the same with the corresponding distribution in guanine, as it can be seen by a comparison of the corresponding wavefunctions in the middle and bottom panels of Fig. 3. Any small differences in the occupation probabilities of these plots reflect the changes in guanine's HOMO or LUMO wavefunctions between the B-DNA and isolated conformations, as it has been discussed above.

It is worth mentioning here that G-C's LUMO wavefunction is the only HOMO or LUMO of both base-pairs which shows a qualitatively different behavior when the base distortions within B-DNA are taken into account, as opposed with the case of the isolated bases. In particular, considering the structure of the isolated bases and calculating A-T's HOMO and LUMO or G-C's HOMO, one finds similar results with the ones presented in this and the previous subsection (for example, A-T's HOMO is localized in A and LUMO in T, while G-C's HOMO is localized in G). On the contrary, application of our method for the G-C LUMO, using the structure of the isolated bases, shows localization mainly in $\mathrm{C}(\approx 80 \%)$ but with a non-vanishing amplitude in $\mathrm{G}(\approx 20 \%)$. In this context note that the isolated $\mathrm{G}$ and $\mathrm{C}$ bases exhibit the same LUMO energy, $-4.4 \mathrm{eV}$ (see Table I). These results seem to be in accordance with the calculations of Ref. [18], although the nature of the HOMO and LUMO base-pair wavefunctions (whether it is of $\pi$ character or not) was not mentioned there. 


\section{B. Charge transfer hopping parameters}

The charge transfer hopping parameters $t_{H}$ and $t_{L}$ are obtained below, as described in Sec. II viz. through the HOMO and LUMO wavefunctions calculated in the previous subsection and Eqs. (5) or (11), for bases or base-pairs, respectively, with $V_{i j}$ given by Eq. (6).

\section{Description at the base-pair level}

At first we present the hopping parameters $t_{H / L}^{b p}$ between successive base-pairs, for the description at the base-pair level discussed in Sec. IIC1 (cf. parameters $t_{H / L}^{b p(\lambda ; \lambda \pm 1)}$ in Eq. (10) $)$. The notation $Y X$ is used here to denote two successive base-pairs, according to the following convention for the DNA strands orientation

$$
\begin{aligned}
& 5^{\prime} \quad 3^{\prime} \\
& Y-Y_{\text {compl }} \\
& X-X_{\text {compl }} \\
& 3^{\prime} \quad 5^{\prime} .
\end{aligned}
$$

$X, X_{\text {compl }}, Y, Y_{\text {compl }}$ denote DNA bases, where $X_{\text {compl }}$ and $Y_{\text {compl }}$ are the complementary bases of $X$ and $Y$, respectively. Therefore, the notation $Y X$ means that the bases $Y$ and $X$ of two successive base-pairs ( $Y-Y_{\text {compl }}$ and $\left.X-X_{\text {compl }}\right)$ are located at the same strand in the direction $5^{\prime}-3^{\prime} . X-X_{\text {compl }}$ is one base-pair of the base-pair dimer and $Y-Y_{\text {compl }}$ is the other base-pair, separated and twisted by $3.14 \AA$ and $36^{\circ}$, respectively, relatively to the first base-pair. For example the notation $\mathrm{AC}$ denotes that the base-pair dimer consists of an adenine-thymine and a cytosine-guanine base-pair, where one strand contains $\mathrm{A}$ and $\mathrm{C}$ in the direction $5^{\prime}-3^{\prime}$ and the complementary strand contains $\mathrm{T}$ and $\mathrm{G}$ in the direction $3^{\prime}-5^{\prime}$.

Table III summarizes the calculated hopping parameters, $t_{H / L}^{b p}$, according to our method, for all possible combinations of successive base-pairs, as well as corresponding values obtained from other theoretical studies. Due to the symmetry between base-pair dimers $Y X$ and $X_{\text {compl }} Y_{\text {compl }}$, the number of different hopping parameters is reduced from sixteen to ten. In Table III basepair dimers exhibiting the same transfer parameters are listed together in the first column. Our predictions for the HOMO, $t_{H}^{b p}$, and LUMO, $t_{L}^{b p}$, hopping parameters are reported in the second and sixth column, respectively. The third column contains the $a b$ initio results of Voityuk et al. 83] for the magnitudes of HOMO transfer parameters. Results from Endres et al. [84] for a few combinations of successive base-pairs, using the densityfunctional-based electronic structure program SIESTA, are presented for $t_{H}^{b p}$ and $t_{L}^{b p}$ in the fourth and seventh columns, respectively. The same authors show similar results in Fig. 4 of Ref. [4]. The corresponding values -approximately extracted from that figure for zero twist angle- are shown in the fifth and eighth columns. As it has been already mentioned, in Ref. [4] the transfer parameters have been obtained using the same theoretical bedrock adopted also in our work -i.e. the Slater-Koster semi-empirical method and Eqs. (6) and (11)- but with slightly different expressions for $V_{p p \pi}$ and $V_{p p \sigma}$.

TABLE III: Base-pair transfer integrals for all possible combinations of successive base-pairs, as shown in the first column according to the notation described in the text, in the direction $5^{\prime}-3^{\prime}$. Hole hopping parameters $t_{H}^{b p}$ obtained from our calculations (through HOMO wavefunctions), from Ref. [83], from Ref. [84], and from Fig. 4 of Ref. [4], are presented in the second, third, fourth, and fifth column, respectively. Electron hopping parameters $t_{L}^{b p}$ obtained from our calculations (through LUMO wavefunctions), from Ref. [84], and from Fig. 4 of Ref. [4], are shown in the sixth, seventh, and eighth column, respectively. These quantities represent the parameters $t_{H / L}^{b p(\lambda ; \lambda \pm 1)}$ which appear in Eq. (10). All hopping integrals $t_{H / L}^{b p}$ are given in $\mathrm{meV}$.

\begin{tabular}{|c|c|c|c|c|c|c|c|}
\hline $\begin{array}{c}\text { Base-pair } \\
\text { sequence }\end{array}$ & $t_{H}^{b p}$ & $\begin{array}{c}\left|t_{H}^{b p}\right| \\
{[83]}\end{array}$ & $\begin{array}{c}t_{H}^{b p} \\
{[84]}\end{array}$ & $\begin{array}{c}t_{H}^{b p} \\
{[4]}\end{array}$ & $t_{L}^{b p}$ & $\begin{array}{c}t_{L}^{b p} \\
{[84]}\end{array}$ & $\begin{array}{c}t_{L}^{b p} \\
{[4]}\end{array}$ \\
\hline \hline AA, TT & -8 & 26 & -70 & $\approx-25$ & -29 & 105 & $\approx 35$ \\
\hline AT & 20 & 50 & & & 0.5 & & \\
\hline AG, CT & -5 & 122 & -71 & $\approx-50$ & 3 & 112 & $\approx 35$ \\
\hline AC, GT & 2 & 27 & & & 32 & & \\
\hline TA & 47 & 55 & & & 2 & & \\
\hline TG, CA & -4 & 26 & & & 17 & & \\
\hline TC, GA & -79 & 25 & -187 & $\approx-160$ & -1 & 47 & $\approx 35$ \\
\hline GG, CC & -62 & 93 & -141 & $\approx-140$ & 20 & 53 & $\approx 35$ \\
\hline GC & 1 & 78 & & & -10 & & \\
\hline CG & -44 & 22 & & & -8 & & \\
\hline
\end{tabular}

We see from Table III that according to our calculations (second column), the higher in magnitude hopping parameters for holes appear in TC, GA, GG, CC, TA, and $\mathrm{CG}$ dimers, where the corresponding transfer matrix elements are around $50 \mathrm{meV}$ or larger. The lower magnitudes of hole hopping parameters (1-2 meV) occur in GC, AC, and GT dimers. According to Ref. [83], the higher values are found in $\mathrm{AG}$ and $\mathrm{CT}$ dimers. In four out of sixteen dimers (GG, CC, CG and TA) the absolute values of our predictions for $t_{H}^{b p}$ and those of Ref. [83] differ by no more than a factor of two, while in other five cases (GC, AC, GT, AG, and CT) our results are significantly smaller by more than one order of magnitude. However, it should be noted that more sophisticated theoretical descriptions [85] have shown that the approximation used in Ref. 83 in general overestimates the transfer integrals. Endres et al. in Refs. [84] and [4] provide $t_{H}^{b p}$ values for a few cases of successive base-pairs, which are larger in magnitude than both our results and those of Ref. [83], with the exception of AG and CT where their values are in between of our results and those of Ref. [83].

Regarding electron hopping parameters (sixth column), we find the highest magnitudes (around $30 \mathrm{meV}$ ) in AC, GT, AA, and TT dimers, while the lowest one is 
in AT (less than $1 \mathrm{meV})$. The most recent $t_{L}^{b p}$ values of Endres et al. in Ref. [4] are closer in magnitude to our predictions, as compared with their former estimates in Ref. 84]. For the majority of base-pair dimers, the hole transfer parameters, as calculated in our work, are larger in magnitude than the corresponding ones for electrons, indicating probably that hole transport is more favorable than electron transport along DNA. This is certainly true for the higher amplitude hopping parameters (larger than $40 \mathrm{meV})$.

\section{Description at the single-base level}

At this level of description a charge carrier is allowed to hop not only between bases belonging to adjacent basepairs, but also from one DNA base to its complementary base within the same base-pair [see the second term in the right-hand-side of Eqs. (13)]. In Table IV we display these interbase intra-base-pair hopping parameters. Our calculations show that the magnitudes of hole and electron transfer integrals within G-C and A-T base-pairs are around $10 \mathrm{meV}$. For holes we find equal hopping parameters within G-C or A-T base-pairs, while for electrons intra-base-pair hopping is more favorable in a $\mathrm{G}-\mathrm{C}$ basepair. First principles results from Ref. [86] show larger magnitudes of electron hoppings than our predictions (almost four times larger), while for holes the magnitudes are larger than ours in A-T and smaller in G-C base-pairs (in both cases the corresponding results differ by a little more than a factor of two).

TABLE IV: Intra-base-pair hopping parameters of the two BDNA base-pairs (first column), for holes $\left(t_{H}^{b}\right.$, second and third column) and electrons ( $t_{L}^{b}$, fourth and fifth column). Our predictions for holes (electrons) are shown in second (fourth) column, while results from Ref. [86] are shown in third (fifth) column, respectively. These quantities represent the parameters $t_{H / L}^{b(\lambda, 1 ; \lambda, 2)}$ and $t_{H / L}^{b(\lambda, 2 ; \lambda, 1)}$ which appear in Eq. (13). All hopping parameters $t_{H / L}^{b}$ are given in $\mathrm{meV}$.

\begin{tabular}{|c|c|c|c|c|}
\hline Base-pair & $t_{H}^{b}$ & $\begin{array}{c}t_{H}^{b} \\
{[86]}\end{array}$ & $t_{L}^{b}$ & $\begin{array}{c}t_{L}^{b} \\
{[86]}\end{array}$ \\
\hline \hline A-T & -12 & 26 & -9 & 34 \\
\hline G-C & -12 & 5 & 16 & 63 \\
\hline
\end{tabular}

Now we turn to interbase transfer integrals between bases of adjacent base-pairs. First we examine intrastrand parameters, i.e. hoppings between successive bases of the same strand [see the third and fourth terms in the right-hand-side of Eqs. (13)]. A similar convention in the notation is used here as previously; $Y X$ implies that the DNA bases $Y$ and $X$ are given in the direction $5^{\prime}-3^{\prime}$ of the strand. For example, the notation TG denotes the strand orientation $5^{\prime}-\mathrm{T}-\mathrm{G}-3^{\prime}$. The intrastrand interbase hopping parameters as obtained from our calculations are presented in Table V] (in second column for holes and eighth for electrons). Regarding hole transfer, the higher in magnitude hopping matrix elements (larger than $100 \mathrm{meV}$ ) are found in TT and CT. Other relatively large magnitudes appear in TC, GC, GA, GT, AT, AC, CC, and GG $\left(\left|t_{H}^{b}\right|>60 \mathrm{meV}\right)$. The lower absolute values of hole hopping parameters correspond to successive $\mathrm{CG}, \mathrm{AG}, \mathrm{CA}$, and AA bases $\left(\left|t_{H}^{b}\right|<10 \mathrm{meV}\right)$. Looking at the electron hopping parameters, the highest magnitudes appear in CT (larger than $60 \mathrm{meV})$ and $\mathrm{CC}, \mathrm{GC}\left(\left|t_{L}^{b}\right|>40 \mathrm{meV}\right)$, while the lower ones in AG, AC, AT, and TA $\left(\left|t_{L}^{b}\right|<10 \mathrm{meV}\right)$. In most of the cases electron hopping parameters are smaller in magnitude than hole transfer parameters between successive bases, similarly with the case of hopping between successive base-pairs.

Other theoretical predictions for intrastrand transfer integrals have been presented in Refs. 85, 86, 87, 88, 89], using methods from first principles. The corresponding values are shown in Table $\mathrm{V}$. Because these methods are usually more reliable for occupied orbitals, there exist many works computing hole hopping parameters $t_{H}^{b}$. In general, our results are in the range of values obtained by these works. The ab initio method used in Ref. [87] (similar to that of Ref. [83]), has been shown to overestimate the transfer parameters [85]. Indeed, in most of the cases the values provided in Ref. [87] are larger than the other $t_{H}^{b}$ values of Table V. Regarding electron hopping parameters, first principles results of $t_{L}^{b}$ for a few cases of successive bases have been presented in Ref. [86], which are in a good agreement with our calculations.

Finally we present interstrand transfer parameters describing "diagonal" interbase hoppings between diagonally located bases of adjacent base-pairs which belong to opposite strands [see the fifth and sixth terms in the right-hand-side of Eqs. (13)]. Here it is necessary to distinguish two sets of diagonal interstrand hoppings, depending on the strand orientation. For the conformation of two successive base-pairs shown in (14), the one set of diagonal interstrand transfer parameters refers to hoppings in the direction $3^{\prime}-3^{\prime}$ (i.e. the path $\left.3^{\prime}-Y_{\text {compl }} \cdots X-3^{\prime}\right)$ and the other set refers to hoppings in the direction $5^{\prime}-5^{\prime}$ (i.e. the path $5^{\prime}-Y \cdots X_{c o m p l}-5^{\prime}$ ).

Table VI shows results for interstrand transfer integrals in the direction $3^{\prime}-3^{\prime}$ for all possible combinations of bases, while Table VII presents corresponding results in the direction $5^{\prime}-5^{\prime}$. Our calculations yield relatively small magnitudes for these matrix elements. In general the obtained values are of the order of meV or tenths of $\mathrm{meV}$, for both electrons and holes, apart from the cases of $3^{\prime}-A \cdots A-3^{\prime}$ and $3^{\prime}-G \cdots G-3^{\prime}$ for holes (exhibiting $\left|t_{H}^{b}\right|$ between $40-50 \mathrm{meV}$ ) and $3^{\prime}-A \cdots A-3^{\prime}$ for electrons (with $t_{L}^{b}$ around $30 \mathrm{meV}$ ). Theoretical predictions for hole interstrand hopping parameters $t_{H}^{b}$ have been also presented for all combinations of bases in Ref. [88]. Other results from first principles methods obtained for a few cases in Refs. [87] (for holes) and [86] (both for electrons and holes), show usually larger magnitudes in comparison to our calculations. 
TABLE V: Intrastrand transfer parameters between two successive DNA bases (first column) in the direction $5^{\prime}-3^{\prime}$ of the strand. Hole hopping parameters $t_{H}^{b}$ obtained from our calculations (through HOMO wavefunctions), and from Refs. [87], 85], 88], 89], and [86], are presented in the second, third, fourth, fifth, sixth, and seventh column, respectively. Electron hopping parameters $t_{L}^{b}$ obtained from our calculations (through LUMO wavefunctions) and from Ref. [86], are shown in the eighth and ninth column, respectively. These quantities represent the parameters $t_{H / L}^{b(\lambda, i ; \lambda \pm 1, i)}$ which appear in Eq. (13). All hopping parameters $t_{H / L}^{b}$ are given in meV.

\begin{tabular}{|c|c|c|c|c|c|c|c|c|}
\hline $\begin{array}{c}\text { Base } \\
\text { sequence }\end{array}$ & $t_{H}^{b}$ & $\mid \begin{array}{c}\left|t_{H}^{b}\right| \\
{[87]}\end{array}$ & $\left|t_{H}^{b}\right|$ & $t_{H}^{b}$ & $\left|t_{H}^{b}\right|$ & $t_{H}^{b}$ & $t_{L}^{b}$ & $t_{L}^{b}$ \\
{$[88]$} & {$[89]$} & {$[86]$} & & {$[86]$} \\
\hline \hline AA & -8 & 30 & 4 & -4 & 8 & 21 & 16 & 25 \\
\hline AT & 68 & 105 & & -63 & 28 & & 7 & \\
\hline AG & -5 & 49 & 44 & -10 & 37 & & 1 & \\
\hline AC & 68 & 61 & & 42 & 30 & & -3 & \\
\hline TA & 26 & 86 & & -31 & 64 & & -7 & \\
\hline TT & -117 & 158 & & 72 & 93 & -98 & -30 & -23 \\
\hline TG & 28 & 85 & 61 & 18 & 70 & & -17 & \\
\hline TC & -86 & 76 & & -28 & 52 & & 22 & \\
\hline GA & -79 & 89 & 36 & -77 & 52 & & 30 & \\
\hline GT & 73 & 137 & 81 & 141 & 49 & & -32 & \\
\hline GG & -62 & 84 & 51 & 53 & 61 & -114 & 20 & 20 \\
\hline GC & 80 & 110 & & -114 & 57 & & 43 & \\
\hline CA & 5 & 29 & & -2 & 5 & & -12 & \\
\hline CT & -107 & 100 & & -55 & 33 & & 63 & \\
\hline CG & -1 & 42 & & 9 & 31 & & 15 & \\
\hline CC & -66 & 41 & & 22 & 26 & -21 & -47 & -60 \\
\hline
\end{tabular}

\section{CONCLUSIONS}

We have systematically studied all the tight-binding parameters which are necessary for the description of charge transfer along DNA. The $\pi$ electronic structure of the four DNA bases (adenine, thymine, cytosine, and guanine) has been calculated by using the LCAO method, employed with a novel parametrization. In addition, we have presented the HOMO and LUMO of the two B-DNA base-pairs (adenine-thymine and guanine-cytosine), using a similar process of linear combination of molecular orbitals. Taking into account the slight deformation of bases within the base-pairs of B-DNA (compared to the isolated bases) we find that (a) for the A-T base-pair the HOMO resides in $\mathrm{A}$ and the LUMO in $\mathrm{T}$, while (b) for the $\mathrm{G}-\mathrm{C}$ base-pair both HOMO and LUMO reside in $\mathrm{G}$.

Our theoretical approach predicts the $\pi$ HOMO energy of DNA bases with a deviation smaller than $0.3 \mathrm{eV}$ in comparison with the experimental values. In particular, for the bases of guanine and cytosine our results coincide with the experimental ones, while for thymine and adenine the difference ranges from $0-0.2 \mathrm{eV}$ and $0.2-0.3 \mathrm{eV}$, respectively. Regarding the first $\pi-\pi^{*}$ transition energy the deviations are larger: $0-0.2 \mathrm{eV}$ for cytosine, 0.4-0.5
TABLE VI: $3^{\prime}-3^{\prime}$ interstrand hopping parameters between two diagonally located DNA bases of adjacent base-pairs (first column). Hole hopping parameters $t_{H}^{b}$ obtained from our calculations (through HOMO wavefunctions) and from Refs. 88], 86], are presented in the second, third, and fourth column, respectively. Electron hopping parameters $t_{L}^{b}$ obtained from our calculations (through LUMO wavefunctions) and from Ref. [86], are shown in the fifth and sixth column, respectively. These quantities represent the parameters $t_{H / L}^{b(\lambda, i ; \lambda \pm 1, j)}$ $(i \neq j)$ which appear in Eq. (13). All hopping parameters $t_{H / L}^{b}$ are given in meV.

\begin{tabular}{|c|c|c|c|c|c|}
\hline $\begin{array}{c}\text { Base } \\
\text { sequence }\end{array}$ & $t_{H}^{b}$ & $\begin{array}{c}t_{H}^{b} \\
{[88]}\end{array}$ & $\left|t_{H}^{b}\right|$ & $t_{L}^{b}$ & $\begin{array}{c}t_{L}^{b} \\
{[86]}\end{array}$ \\
\hline \hline AA & 48 & 49 & & 29 & \\
\hline AT, TA & -3 & -7 & 9 & 3 & -13 \\
\hline AG, GA & -3 & -11 & & -6 & \\
\hline AC, CA & -5 & 17 & & -3 & \\
\hline TT & 0.5 & 6 & & 0.2 & \\
\hline TG, GT & 5 & -14 & & 2 & \\
\hline TC, CT & 0.5 & 4 & & -0.2 & \\
\hline GG & -44 & -32 & & -5 & \\
\hline GC, CG & 4 & 22 & 48 & -4 & -15 \\
\hline CC & 1 & 10 & & 0.3 & \\
\hline
\end{tabular}

TABLE VII: $5^{\prime}-5^{\prime}$ interstrand hopping parameters between two diagonally located DNA bases of adjacent base-pairs (first column). Hole hopping parameters $t_{H}^{b}$ obtained from our calculations (through HOMO wavefunctions) and from Refs. [88], 87], 86], are presented in the second, third, fourth, and fifth column, respectively. Electron hopping parameters $t_{L}^{b}$ obtained from our calculations (through LUMO wavefunctions) and from Ref. [86] are shown in the sixth and seventh column, respectively. These quantities represent the parameters $t_{H / L}^{b(\lambda, i ; \lambda \pm 1, j)}(i \neq j)$ which appear in Eq. (13). All hopping parameters $t_{H / L}^{b}$ are given in $\mathrm{meV}$.

\begin{tabular}{|c|c|c|c|c|c|c|}
\hline $\begin{array}{c}\text { Base } \\
\text { sequence }\end{array}$ & $t_{H}^{b}$ & $\begin{array}{c}t_{H}^{b} \\
{[88]}\end{array}$ & $\begin{array}{c}\left|t_{H}^{b}\right| \\
{[87]}\end{array}$ & $\begin{array}{c}t_{H}^{b} \\
{[86}\end{array}$ & $t_{L}^{b}$ & $\left.\begin{array}{c}t_{L}^{b} \\
{[86}\end{array}\right]$ \\
\hline \hline AA & 2 & 31 & $31-35$ & & 6 & \\
\hline AT, TA & 9 & 7 & $16-20$ & -11 & 2 & -10 \\
\hline AG, GA & 4 & -13 & $19-24$ & & 3 & \\
\hline AC, CA & 5 & -1 & & & -2 & \\
\hline TT & 4 & 1 & $3-4$ & & 2 & \\
\hline TG, GT & 5 & -9 & & & 3 & \\
\hline TC, CT & 2 & 0.3 & & & -2 & \\
\hline GG & 3 & 12 & 19 & & -2 & \\
\hline GC, CG & 4 & 2 & & -8 & -3 & -12 \\
\hline CC & 1 & 1 & 1 & & 2 & \\
\hline
\end{tabular}

$\mathrm{eV}$ for thymine, 0.5-0.7 eV for guanine, and 0.7-1.0 eV for adenine. Our results for the HOMO and LUMO energies and wavefunctions of bases and also of base-pairs have been compared with other theoretical calculations, 
using methods from first principles.

We provide estimates for the complete set of hopping parameters (both for electrons and holes) between successive base-pairs and between neighboring bases (i.e., successive bases in the same strand, complementary bases within a base-pair, and adjacent base-pairs' diagonally located bases in opposite strands) in B-DNA, including all possible combinations of them. Our predictions for the transfer parameters are compared with other theoretical estimates, when available, and in most cases there is an agreement in the order of magnitude of the results. In general, the hopping parameters for hole transfer obtained through the HOMO wavefunctions, are higher in magnitude compared to the ones for electron transfer obtained through the LUMO wavefunctions. These theoretical calculations show that probably hole transport along DNA is more favorable than electron transport.

The microscopic quantities calculated in this work, i.e. the HOMO and LUMO energies as well as the hopping matrix elements, provide all necessary parameters for a tight-binding phenomenological description of charge transfer along the DNA double helix, based either on bases' or base-pairs' $\pi$ molecular overlap. Taking advantage of such a description, at a mesoscopic level, the temporal and spatial evolution of electron or hole transport along DNA can be examined in experimentally relevant time and length scales. Furthermore, important measurable quantities like hole/electron transmission coefficients and conductivities can be calculated for any DNA segment (of whatever base sequence) under consideration, since there are well-established techniques to compute such a quantities from tight-binding models.

Acknowledgements We acknowledge useful discussions with N. Lathiotakis and support from the C. Carathéodory program C155 of University of Patras.
[1] C. Dekker and M. Ratner, Phys. World, August 2001, 29 (2001).

[2] K. Keren, M. Krueger, R. Gilad, G. Ben-Yoseph, U. Sivan, and E. Braun, Science 297, 72 (2002).

[3] N.C. Seeman, Nature 421, 427 (2003).

[4] R.G. Endres, D.L. Cox, and R.R.P. Singh, Rev. Mod. Phys. 76, 195 (2004).

[5] C.J. Burrows and J.G. Muller, Chem. Rev. 98, 1109 (1998).

[6] J. Cadet in DNA Adducts: Identification and Significance, eds. K. Hemminki, A. Dipple, D.E.F. Shiker, F.F. Kadlubar, D. Segerback, and H. Bartsch, IARC, Lyon (1994).

[7] P.J. Dandliker, R.E. Holmlin, J.K. Barton, Science 275, 1465 (1997).

[8] S.R. Rajski, B.A. Jackson, and J.K. Barton, Mutation Research 447, 49 (2000).

[9] Y. Zhang, R.H. Austin, J. Kraeft, E.C. Cox, and N.P. Ong, Phys. Rev. Lett. 89, 198102 (2002).

[10] A.J. Storm, J. van Noort, S. de Vries, and C. Dekker, Appl. Phys. Lett. 79, 3881 (2001).

[11] P.J. de Pablo, F. Moreno-Herrero, J. Colchero, J. Gómez Herrero, P. Herrero, A.M. Baró, P. Ordejón, J.M. Soler, and E. Artacho, Phys. Rev. Lett. 85, 4992 (2000).

[12] K.-H. Yoo, D.H. Ha, J.-O. Lee, J.W. Park, Jinhee Kim, J.J. Kim, H.-Y. Lee, T. Kawai, and Han Yong Choi, Phys. Rev. Lett. 87, 198102 (2001).

[13] L. Cai, H. Tabata, and T. Kawai, Appl. Phys. Lett. 77, 3105 (2000).

[14] H.W. Fink and C. Schonenberger, Nature 398, 407 (1999).

[15] P. Tran, B. Alavi, and G. Gruner, Phys. Rev. Lett. 85, 1564 (2000).

[16] Z. Kutnjak, C. Filipič, R. Podgornik, L. Nordenskiöld, and N. Korolev, Phys. Rev. Lett. 90, 098101 (2003).

[17] J.B. MacNaughton, A. Moewes, J.S. Lee, S.D. Wettig, H.-B. Kraatz, L.Z. Ouyang, W.Y. Ching, and E.Z. Kurmaev, J. Phys. Chem. B 110, 15742 (2006).

[18] S.S. Mallajosyula, A. Datta, and S.K. Pati, Synth. Met. 155, 398 (2005).
[19] P. Maragakis, R.L. Barnett, E. Kaxiras, M. Elstner, and T. Frauenheim, Phys. Rev. B 66, 241104 (2002).

[20] A. Rakitin, P. Aich, C. Papadopoulos, Yu. Kobzar, A.S. Vedeneev, J.S. Lee, and J.M. Xu, Phys. Rev. Lett. 86, 3670 (2001).

[21] M. Fuentes-Cabrera, B.G. Sumpter, and J.C. Wells, J. Phys. Chem. B 109, 21135 (2005); M. Fuentes-Cabrera, B.G. Sumpter, P. Lipkowski, and J.C. Wells, J. Phys. Chem. B 110, 6379 (2006); M. Fuentes-Cabrera, X. Zhao, P.C. Kent, and B.G. Sumpter, J. Phys. Chem. B 111, 9057 (2007).

[22] M.R. Arkin, E.D.A. Stemp, R.E. Holmlin, J.K. Barton, A. Hörmann, E.J.C. Olson, and P.F. Barbara, Science 273, 475 (1996); D.B. Hall, R.E. Holmlin, and J.K. Barton, Nature 382, 731 (1996).

[23] D. Porath, A. Bezryadin, S. de Vries, and C. Dekker, Nature 403, 635 (2000).

[24] Ch. Adesi, S. Walch, and M.P. Anantram, Phys. Rev. B 67, 081405 (R) (2003); Ch. Adesi and M.P. Anantram, Appl. Phys. Lett. 82, 2353 (2003).

[25] S.V. Rakhmanova and E.M. Conwell, J. Phys. Chem. B 105, 2056 (2001).

[26] W. Zhang, A.O. Govorov, and S.E. Ulloa, Phys. Rev. B 66, 060303 (2002).

[27] S. Komineas, G. Kalosakas, and A.R. Bishop, Phys. Rev. E 65, 061905 (2002); P. Maniadis, G. Kalosakas, K.Ø. Rasmussen, and A.R. Bishop, Phys. Rev. B 68, 174304 (2003).

[28] M.R. Singh, J. Biomater. Sci. Polymer Edn. 15, 1533 (2004).

[29] G.P. Triberis, C. Simserides, and V.C. Karavolas, J. Phys.: Condens. Matter 17, 2681 (2005); G.P. Triberis and M. Dimakogianni, J. Phys.: Condens. Matter 21, 035114 (2009).

[30] Y.-J. Ye, R.-S. Chen, A. Martinez, P. Otto, J. Ladik, Solid State Commun. 112, 139 (1999).

[31] P.T. Henderson, D. Jones, G. Hampikian, Y. Kan, and G.B. Schuster, Proc. Natl. Acad. Sci. USA 96, 8353 (1999).

[32] R. Bruinsma, G. Grüner, M.R. D'Orsogna, and J. Rud- 
nick, Phys. Rev. Lett. 85, 4393 (2000).

[33] G. Kalosakas, K.Ø. Rasmussen, and A.R. Bishop, J. Chem. Phys. 118, 3731 (2003); Synth. Met. 141, 93 (2004).

[34] Z.G. Yu and X. Song, Phys. Rev. Lett. 86, 6018 (2001).

[35] D. Hennig, Eur. Phys. J. B 30, 211 (2002); D. Hennig, E.B. Starikov, J.F.R. Archilla, and F. Palmero, J. Biol. Phys. 30, 227 (2004)

[36] G. Kalosakas, K.L. Ngai, and S. Flach, Phys. Rev. E 71, 061901 (2005); P. Maniadis, G. Kalosakas, K.Ø. Rasmussen, and A.R. Bishop, Phys. Rev. E 72, 021912 (2005).

[37] E. Diaz, R.P.A. Lima, and F. Dominguez-Adame, Phys. Rev. B 78, 134303 (2008).

[38] L.G.D. Hawke, G. Kalosakas, and C. Simserides, Mol. Phys. 107, 1755 (2009).

[39] L.G.D. Hawke, C. Simserides, and G. Kalosakas, Mater. Sci. Eng. B, in press (2009), doi:10.1016/j.mseb.2009.02.012

[40] W.A. Harrison, Electronic structure and the properties of solids, 2nd edition, Dover, New York (1989); Elementary electronic structure, World Scientific (1999).

[41] J.C. Slater and G.F. Koster, Phys. Rev. 94, 1498 (1954).

[42] M. Menon and R.E. Allen, Phys. Rev. B 38, 6196 (1988).

[43] N. Lathiotakis and A.N. Andriotis, Solid State Comm. 87, 871 (1993); M. Menon, J. Connolly, N. Lathiotakis, and A. Andriotis, Phys. Rev. B 50, 8903 (1994).

[44] http://chemistry.gsu.edu/Glactone/PDB/pdb.html

[45] N.S. Hush and A.S. Cheung, Chem. Phys. Lett. 34, 11 (1975).

[46] G. Lauer, W. Schafer, and A. Schweig, Tetrahedron Lett. 45, 3939 (1975).

[47] D. Dougherty, K. Wittel, J. Meeks, and S.P. McGlynn, J. Am. Chem. Soc. 98, 3815 (1976).

[48] J. Lin, C. Yu, S. Peng, I. Akiyama, K. Li, L.K. Lee, P.R. LeBreton, J. Am. Chem. Soc. 102, 4627 (1980); J. Lin, C. Yu, S. Peng, I. Akiyama, K. Li, L.K. Lee, P.R. LeBreton, J. Phys. Chem. 84, 1006 (1980).

[49] S. Urano, X. Yang, and P. R. LeBreton, Journal of Molecular Sructure 214, 315 (1989).

[50] D. Voet, W.B. Gratzer, R.A. Cox, and P. Doty, Biopolymers 1, 193 (1963).

[51] L.B. Clark, G.G. Peschel, and I. Tinoco Jr., J. Phys. Chem. 69, 3615 (1965).

[52] D.W. Miles, R.K. Robins, and H. Eyring, Proc. Natl. Acad. Sci. USA 57, 1139 (1967).

[53] T. Yamada and H. Fukutome, Biopolymers 6, 43 (1968).

[54] W. Voelter, R. Records, E. Bunnenberg, and C. Djerassi, J. Am. Chem. Soc. 90, 6163 (1968).

[55] A.F. Fucaloro and L.S. Forster, J. Am. Chem. Soc. 93, 6443 (1971).

[56] W.C. Brunner and M.F. Maestre, Biopolymers 14, 555 (1975).

[57] C.A. Sprecher and W. C. Johnson, Biopolymers 16, 2243 (1977).

[58] K. Raksanyi, I. Foldvary, J. Fidy, and L. Kittler, Biopolymers 17, 887 (1978).

[59] Y. Matsuoka and B. Norden, J. Phys. Chem. 86, 1378 (1982).

[60] F. Zaloudek, J.S. Novros, and L.B. Clark, J. Am. Chem.
Soc. 107, 7344 (1985).

[61] L.B. Clark, J. Phys. Chem. 94, 2873 (1990).

[62] A. Holmen, A. Broo, B. Albinsson, and B. Norden, J. Am. Chem. Soc. 119, 12240 (1997).

[63] A.O. Colson, B. Besler, D.M. Close, and M.D. Sevilla, J. Phys. Chem. 96, 661 (1992).

[64] H. Sugiyama and I. Saito, J. Am. Chem. Soc. 118, 7063 (1996).

[65] M. Hutter and T. Clark, J. Am. Chem. Soc. 118, 7574 (1996).

[66] M.P. Fülscher and B.O. Roos, J. Am. Chem. Soc. 117, 2089 (1995).

[67] J. Lorentzon, M.P. Fülscher, and B.O. Roos, J. Am. Chem. Soc. 117, 9265 (1995).

[68] M.P. Fülscher, L. Serrano-Andres, and B.O. Roos, J. Am. Chem. Soc. 119, 6168 (1997).

[69] M.K. Shukla and P.C. Mishra, Chem. Phys. 240, 319 (1999).

[70] B. Mennucci, A. Toniolo, and J. Tomasi, J. Phys. Chem. A. 105, 4749 (2001).

[71] X. Li, Z. Cai, and M.D. Sevilla, J. Phys. Chem. B 105 , 10115 (2001); X. Li, Z. Cai, and M.D. Sevilla, J. Phys. Chem. A 106, 9345 (2002).

[72] A.L. Sobolewski and W. Domcke, Eur. Phys. J. D 20, 369 (2002).

[73] N. Ismail, L. Blancafort, M. Olivucci, B. Kohler, and M.A. Robb, J. Am. Chem. Soc. 124, 6818 (2002).

[74] M.K. Shukla and J. Leszczynski, J. Phys. Chem. A. 106, 11338 (2002).

[75] M.K. Shukla and J. Leszczynski, Journal of Computational Chemistry 25, 768 (2004).

[76] D. Varsano, R. Di Felice, M.A.L. Marques, and A. Rubio, J. Phys. Chem. B 110, 7129 (2006).

[77] T. Fleig, S. Knecht, and C. Hättig, J. Phys. Chem. A 111, 5482 (2007)

[78] H. Zhang, X.Q. Li, P. Ham, X.Y. Yu, and Y.J. Yan, J. Chem. Phys. 117, 4578 (2002).

[79] M.K. Shukla and J. Leszczynski, J. Phys. Chem. A 106, 4709 (2002).

[80] http://www.jmol.org/

[81] T. Cramer, S. Krapf, and T. Koslowski, J. Phys. Chem. B 108, 11812 (2004).

[82] R. Di Felice, A. Calzolari, and E. Molinari, Phys. Rev. B 65, 045104 (2001).

[83] A.A. Voityuk, J. Jortner, M. Bixon, and N. Rösch, J. Chem. Phys. 114, 5614 (2001).

[84] R.G. Endres, D.L. Cox, and R.R.P. Singh, arXiv:cond-mat/0201404 v2 (2002).

[85] L. Blancafort and A.A. Voityuk, J. Phys. Chem. A 110 , 6426 (2006).

[86] H. Mehrez and M.P. Anantram, Phys. Rev. B 71, 115405 (2005).

[87] A.A. Voityuk, N. Rösch, M. Bixon, and J. Jortner, J. Phys. Chem. B 104, 9740 (2000).

[88] K. Senthilkumar, F.C. Grozema, C.F. Guerra, F.M. Bickelhaupt, F.D. Lewis, Y.A. Berlin, M.A. Ratner, and L.D. A. Siebbeles, J. Am. Chem. Soc. 127, 14894 (2005).

[89] T. Kubar, P.B. Woiczikowski, G. Cuniberti, and M. Elstner, J. Phys. Chem. B 112, 7937 (2008). 